\title{
Weighted directed clustering: Interpretations and requirements for heterogeneous, inferred, and measured networks
}

\author{
Tanguy Fardet $\odot$ and Anna Levina $\odot$ \\ University of Tübingen, Tübingen 72076, Germany \\ and Max Planck Institute for Biological Cybernetics, Tübingen 72076, Germany
}

(Received 8 June 2021; accepted 18 October 2021; published 17 November 2021)

\begin{abstract}
Weights and directionality of the edges carry a large part of the information we can extract from a complex network. However, many network measures were formulated initially for undirected binary networks. The necessity to incorporate information about the weights led to the conception of multiple extensions, particularly for definitions of the local clustering coefficient discussed here. We uncover that not all of these extensions are fully weighted; some depend on the degree and thus change a lot when an infinitely-small-weight edge is exchanged for the absence of an edge, a feature that is not always desirable. We call these methods "hybrid" and argue that, in many situations, one should prefer fully weighted definitions. After listing the necessary requirements for a method to analyze many various weighted networks properly, we propose a fully weighted continuous clustering coefficient that satisfies all the previously proposed criteria while also being continuous with respect to vanishing weights. We demonstrate that the behavior and meaning of the Zhang-Horvath clustering and our proposed continuous definition provide complementary results and significantly outperform other definitions in multiple relevant conditions. We demonstrate, using synthetic and real-world networks, that when the network is inferred, noisy, or very heterogeneous, it is essential to use the fully weighted clustering definitions.
\end{abstract}

DOI: 10.1103/PhysRevResearch.3.043124

\section{INTRODUCTION}

The clustering coefficient (CC) was originally introduced for binary undirected networks to quantify strong connectedness within a local neighborhood. It was defined as the fraction of all possible triangles that were realized, i.e., the ratio between all triangles in which node $i$ participates $\left(n_{\Delta, i}\right)$ and the total number of triangles that could theoretically be made given its degree $d_{i}$, which is the number of triplets $\left(n_{T, i}\right)$ :

$$
C_{i}^{\text {bin }}=\frac{n_{\Delta, i}}{n_{T, i}}=\frac{n_{\Delta, i}}{d_{i}\left(d_{i}-1\right)} .
$$

From a neighbor-centric perspective, it can be seen perhaps more intuitively as the probability that two neighbors of a node are connected. However, as network science expanded, more and more graphs were encountered, where directedness and edge weights play a central role. Generalizations of the clustering coefficient were therefore introduced to account for asymmetry in the connections between pairs of nodes or heterogeneity in their strength.

The importance of clustering, including its directed variants, to understand complex dynamics on networks has been

Published by the American Physical Society under the terms of the Creative Commons Attribution 4.0 International license. Further distribution of this work must maintain attribution to the author(s) and the published article's title, journal citation, and DOI. Open access publication funded by the Max Planck Society. stressed in multiple studies [1-4]. This is notably the case for the middleman motif, which is a marker of feedforward loops in transcriptional networks and of information transfer redundancy, e.g., in neuroscience. More generally, such motifs will influence the evolution of dynamical processes on the networks, for instance, synchronization patterns, and have been shown to characterize families of networks such as transcription or language networks [2]. Finally, clustering is used in other measurements to access the small-world propensity of networks [5], and the choice of a specific definition can therefore influence whether the network of interest will register as small world or not.

In many applications, network topology and weights are measured only up to a certain precision [6,7]. For example, in neuroscience, the functional connectivity networks are measured using the indirect inference of connections from the recorded activity $[8,9]$. Accepting the inevitability of noise in a network brings forward new requirements on the network measures, namely, that they are stable to the noise and do not change dramatically if the weights are perturbed or weak connections are randomly omitted.

There is no agreement among researchers as to which weighted extension of the clustering coefficient definition is most appropriate. The three predominantly used methods at the moment [10-12] differ in many properties of their definitions. Part of the reason for the absence of a single best weighted clustering lies in a different interpretation of weights in various data sets. Consequently, a different weighted extension might be most appropriate for various data and specific scientific questions. However, to understand which method to 
use when and why, we need to understand their differences precisely.

The difficulty of extending graph measures to weighted networks is not specific to the clustering coefficient but can occur whenever ratios of degrees or path length are involved. We will therefore also discuss a second clustering-related measure, called the closure coefficient and introduced as the fraction of all open walks of length 2 starting from node $i$ that are part of a triangle [13]. This will also enable us to discuss the complementarity of closure and clustering as the former provides an important complement to analyze the tendency of nodes to form 3- and 4-cliques.

We introduce here a distinction between fully weighted and hybrid definitions and discuss why, for several classes of networks, fully weighted and directed definitions should be preferred to other clustering definitions that are currently used for network analysis. We also propose a definition that obeys additional conditions, including continuity of the results with respect to infinitesimal changes in edge weights, which has significant consequences for the resilience to noise in inferred networks. We demonstrate why fully weighted methods are essential for measured and inferred networks, which are pervasive in biological fields such as neuroscience, and for networks dealing with flows of information, money, or goods that display a very broad weight distribution.

\section{INTERPRETATION AND PURPOSE OF WEIGHTED CLUSTERING}

\section{A. Desired properties of weighted clustering coefficients}

Weighted measures are crucial for many network types where the binary connectivity is either uninformative (fully connected network) or displays similar or lower heterogeneity compared with the weighted structure. In this paper, we focus on two classes of real-world networks: inferred or measured networks where there can be a large number of spurious (false positive) edges with small weights; and networks associated with flows of information or goods, which often display broad weight distributions. This is notably the case for many networks in neuroscience, and more generally in information, transportation, or other social and economic networks. Weights are essential to understand the dynamical processes that occur in these networks, requiring measures that go beyond the binary structure.

There could be multiple requirements for weighted clustering coefficients [14] depending on the particular question of interest and on the network properties. The main requirements that we considered necessary for a weighted clustering coefficient are (i) normalization $\left(C_{i} \in[0,1]\right.$ ), (ii) consistency with the binary definition (for binary networks, it should give back the classical result), (iii) linearity (scaling by $\alpha$ all edges involving node $i$ and all edges in triangles including node $i$ scales $C_{i}$ by $\alpha$ ), and (iv) continuity (weak influence of the addition or deletion of edges having very small weights, meaning that an edge with infinitesimally small weight should be equivalent to the absence of that edge).

Compared with a previously proposed list of conditions given by Saramäki et al. [14], we added a continuity condition but did not include a requirement of a specific normalization factor [the global $\max (w)$ ] as long as the normalization condition is fulfilled since only the normalization matters. We omitted the last two conditions of Saramäki et al.'s paper (invariance to weight permutation and ignorance of weights not participating in any triangle). Although they might be of interest for some specific applications, we do not consider them to be generally desired properties for a clustering coefficient. Indeed, the clustering coefficient is a node-centric measure, so there is no reason to expect invariance when the weights of a node's edges change. As for edge ignorance, in the original classifying paper [14] this property is introduced as a particular feature of the Onnela et al. [11] definition, without reasons why it may be a desired condition. We also did not require that all weights in a triangle should be accounted for because this condition is necessarily met if the continuity condition is fulfilled.

Continuity can be expressed mathematically as follows: for a graph $G(V, E)$, if a weighted edge $(u, v, w)$ with $u, v \in V$ and weight $w \in \mathbb{R}$ is added to this graph to form a new graph $G^{\prime}\left(V, E^{\prime}\right)$, with $E^{\prime}=E+\{(u, v, w)\}$, then the clustering measure is continuous if and only if $\forall i \in V, C_{i}^{\left(G^{\prime}\right)} \underset{w \rightarrow 0^{+}}{\longrightarrow} C_{i}^{(G)}$. This condition in crucial to ensure a reasonable behavior of the clustering coefficient in inferred networks.

Though some definitions of previously proposed weighted clustering coefficient definitions obey most of the required properties, none of them completely fulfill the continuity condition-despite previous claims [15]. This is why we will later propose a definition that fulfills all aforementioned conditions. An extensive comparison of the properties fulfilled by different clustering definitions can be found in Appendix B.

\section{B. State of the art for weighted clustering}

First we introduce and classify the existing weighted clustering coefficient definition. For all clustering definitions in the main text, we use the following notation: $A$ is an adjacency matrix, and $W=\left\{w_{i j}\right\}$ is the normalized weight matrix, obtained from the original weight matrix $\tilde{W}=\left\{\tilde{w}_{i j}\right\}$ by $w_{i j}=$ $\tilde{w}_{i j} / \max _{i, j}\left(\tilde{w}_{i j}\right)$.

Hybrid definitions were the first extensions of the binary clustering coefficient definitions. They combine properties associated with a weighted connectivity matrix (i.e., the intensity of the triangle) with properties that could be already obtained from an adjacency matrix (i.e., node degrees). These definitions move from an integer counting the number of triangles $\left(n_{\Delta}\right)$ to a sum of real numbers (computed as a function of edge weights) that we call "intensities" of triangles $\left(I_{\Delta}\right)$. The choice of a particular function for the intensity of the triangles determines the properties of the clustering coefficient.

Two popular hybrid weighted clusterings were given by Barrat et al. [10] and Onnela et al. [11], which we will refer to as the Barrat and Onnela definitions, respectively.

For a node $i$ in a graph, the Barrat definition [10] quantifies the fraction of the node's strength that is invested in triangles (see Appendix C 1 for more details):

$$
C_{i}^{B}=\frac{\left(W A^{2}\right)_{i i}}{2 s_{i}\left(d_{i}-1\right)}=C_{i}^{\mathrm{bin}} \frac{\overline{w_{i}^{\Delta}}}{\overline{w_{i}}}
$$


where $s_{i}=\sum_{j \neq i} w_{i j}$ is the strength of the node, $\overline{w_{i}}$ is the average weight of the edges involving $i$, and $\overline{w_{i}^{\Delta}}=$ $\sum_{j \neq k} \frac{w_{i j}+w_{i k}}{2 n_{\Delta, i}} a_{i j} a_{i k} a_{j k}$ is the average weight of edges involving $i$ that are part of a triangle computed over all triangles in which node $i$ participates. In terms of triangle intensity, this definition was originally written as

$$
\begin{aligned}
C_{i}^{B} & =\frac{\sum_{j \neq k} \frac{w_{i j}+w_{i k}}{2} a_{i j} a_{i k} a_{j k}}{2 s_{i}\left(d_{i}-1\right)} \\
& =\frac{1}{d_{i}\left(d_{i}-1\right)} \sum_{j \neq k} \frac{w_{i j}+w_{i k}}{2 \overline{w_{i}}} a_{i j} a_{i k} a_{j k},
\end{aligned}
$$

thus defining the intensity of triangle $\Delta_{i j k}$ as $I_{\Delta i j k}^{B}=$ $\frac{w_{i j}+w_{i k}}{2 \bar{w}_{i}} a_{i j} a_{i k} a_{j k}$ as the function of two of the triangle's weights and the average weight of the edges connected to node $i, \overline{w_{i}}$.

Proposed a bit later, the Onnela definition [11] scales the binary clustering by the average intensity of the triangles (see Appendix C 2 for more details):

$$
C_{i}^{O}=\frac{\left(W\left[\frac{1}{3}\right]\right)_{i i}^{3}}{d_{i}\left(d_{i}-1\right)}=C_{i}^{\mathrm{bin}} \overline{I_{\Delta i j k}^{O}}
$$

with the triangle intensity defined as $I_{\Delta i j k}^{O}=\left(w_{i j} w_{i k} w_{j k}\right)^{1 / 3}$ and the average intensity taken over all triangles in which $i$ participates.

For all hybrid methods the denominator relies on the node's degree, meaning that the addition or deletion of edges will always significantly affect the clustering coefficient even if the edge has an infinitely small weight. Such methods can thus lead to inaccurate results when applied to the inferred networks, where a significant fraction of edges are false positives with small weights. In the following we will also demonstrate that they cannot reliably detect the most strongly clustered nodes in structured networks. Only fully weighted definitions can rise up to these challenges.

Fully weighted definitions are variants of the clustering coefficient that do not include any binary measures (anything that can be derived from the adjacency matrix alone, e.g., degrees). In addition to substituting the number of triangles by the sum of triangle intensities, they also move away from counting triplets-defining the maximum number of possible triangles $\max \left(n_{\Delta, i}\right)=n_{T, i}=d_{i}\left(d_{i}-1\right)$. Instead, they introduce the triplet intensity $I_{T}$ such that, for a node $i, I_{T, i}$ is a real-valued function of the weights associated with $i$.

One of the first fully weighted definitions for the clustering coefficient was provided by Zhang and Horvath [12] to analyze gene coexpression networks:

$$
C_{i}^{Z}=\frac{\sum_{j \neq k} w_{i j} w_{i k} w_{j k}}{\sum_{j \neq k} w_{i j} w_{i k}}=\frac{\overline{I_{\Delta i j k}^{Z}}}{\overline{I_{T i j k}^{Z}}} C_{i}^{\text {bin }},
$$

which we will refer to as the Zhang-Horvath definition. Note that the fact that the definition can be expressed as a function of the binary clustering does not contradict the fully weighted nature of the measure, as it stems from a simple recombination of the terms.

This definition can be interpreted as the ratio of the summed intensities $I_{\Delta i j k}^{Z}=w_{i j} w_{i k} w_{j k}$ of the triangles $\Delta_{i j k}=(i, j, k)$ to the maximum possible summed intensities
TABLE I. Limit values for undirected weighted clustering coefficients of vertex $i$ (solid circles) for different weight configurations in graphs with vanishing weights. Solid lines depict edges of weight $w=\max (w)=1$, and dotted lines denote edges with vanishing weight $\epsilon$. Only the proposed continuous clustering (bottom row)

\begin{tabular}{|c|c|c|c|c|c|c|c|c|}
\hline & & & & & & & & 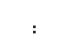 \\
\hline$C^{B}$ & 1 & 1 & 1 & 1 & $1 / 2$ & 0 & $1 / 3$ & $1 / 2$ \\
\hline$C^{O}$ & 0 & 0 & 0 & 0 & $1 / 3$ & 0 & 0 & 0 \\
\hline$C^{Z}$ & 0 & 1 & 0 & 1 & 1 & 0 & $1 / 3$ & 0 \\
\hline$C$ & 0 & 0 & 0 & 0 & 1 & 0 & 0 & 0 \\
\hline
\end{tabular}
returns values consistent with the continuity condition, whereas the Barrat $\left(C^{B}\right)$, Onnela $\left(C^{O}\right)$, and Zhang-Horvath definitions $\left(C^{Z}\right)$ deviate for it.

$I_{\Delta i j k}^{Z(\max )}=I_{T i j k}^{Z}=w_{i j} w_{i k}$ if all existing triplets $T_{i j k}$ were closed by an edge of weight 1 (the maximum possible weight in the normalized network). This way, if $i$ is involved in a single triangle, the clustering coefficient is equal to the weight of the edge closing the triplet centered on $i$ (see also Table I).

Though this definition does not fulfill the continuity property, we will show that it still provides a consistent interpretation of weighted clustering, as discussed in Ref. [16], and is well suited to tackle networks with a large fraction of false positives.

Other fully weighted definitions that have been proposed and discussed since $[15,17,18]$ do not bring significant additions compared with the Zhang-Horvath definition while actually losing some of its properties and its straightforward interpretation. They are not considered further in this paper; see Appendix A for further explanations.

\section{A continuous definition for weighted clustering and closure}

For an undirected graph $G$, we define the proposed continuous clustering of node $i$ as

$$
C_{i}=\frac{\sum_{j \neq k} I_{\Delta i j k}^{2}}{\sum_{j \neq k} I_{T i j k}}=\frac{\sum_{j \neq k}\left(\sqrt[3]{w_{i j} w_{j k} w_{i k}}\right)^{2}}{\sum_{j \neq k} \sqrt{w_{j i} w_{i k}}} .
$$

We define the weighted intensity of triangles and triplets, $I_{\Delta i j k}=\sqrt[3]{w_{i j} w_{j k} w_{i k}}$ and $I_{T i j k}=\sqrt{w_{j i} w_{i k}}$, respectively, using the geometric mean of the weights involved. Because of this, one strong weight in a triangle or triplet cannot compensate the presence of smaller weights, in contrast to what may happen if one uses the arithmetic mean. This provides the desired property that the intensity of triangles and triplets will go to zero if even a single edge weight goes to zero. Note that, though the triangle intensity is defined as the geometric mean of the three weights involved, it is the square of this intensity that is used in the definition. The reason for this choice is twofold: to assign higher influence to large triangles and to ensure the linearity of the coefficient. Importantly, this definition of the triangle intensity $I_{\Delta}$ assigns the same role to all participating edges. The proposed clustering could be interpreted as the ratio of the triangle intensity that is invested in strong triangles (given by the sum of the squared intensities of triangles, which increases the importance of strong triangles) to the triplet intensity, which would represent the maximum 
TABLE II. Definitions of the continuous intensities for each partial mode pattern in directed graphs. Column 1, pattern names; column 2, pattern illustration; column 3, number of triangles for node $i$; column 4, number of triplets for node $i$; column 5 , continuous intensities of the triangles for node $i$; and column 6, continuous intensities of triplets for node $i$. The clustering coefficients associated with each mode $m$ are given by $C_{i}^{(m) \text { bin }}=n_{\Delta, i}^{(m)} / n_{T, i}^{(m)}$ for binary networks and $C_{i}^{(m)}=I_{\Delta, i}^{(m)} / I_{T, i}^{(m)}$ for the continuous definition.

\begin{tabular}{|c|c|c|c|c|c|}
\hline Mode & Pattern & $n_{\Delta, i}^{(m)}$ & $n_{T, i}^{(m)}$ & $I_{\Delta, i}^{(m)}$ & $I_{T, i}^{(m)}$ \\
\hline Cycle & & $A_{i i}^{3}$ & $\begin{array}{l}d_{i, \text { in }} d_{i, \text { out }}-d_{i}^{\leftrightarrow} \\
=\sum_{j \neq k} a_{i j} a_{k i}\end{array}$ & $\left(W^{\left[\frac{2}{3}\right]}\right)_{i i}^{3}$ & $s_{i, \text { in }}^{\left[\frac{1}{2}\right]} s_{i, \text { out }}^{\left[\frac{1}{2}\right]}-s_{i}^{\leftrightarrow}$ \\
\hline Middleman & & $\left(A A^{T} A\right)_{i i}$ & $\begin{array}{l}d_{i, i n} d_{i, \text { out }}-d_{i}^{\leftrightarrow} \\
=\sum_{j \neq k} a_{i j} a_{k i}\end{array}$ & $\left(W^{\left[\frac{2}{3}\right]} W^{\left[\frac{2}{3}\right] T} W^{\left[\frac{2}{3}\right]}\right)_{i i}$ & $s_{i, \text { in }}^{\left[\frac{1}{2}\right]} s_{i, \text { out }}^{\left[\frac{1}{2}\right]}-s_{i}^{\leftrightarrow}$ \\
\hline Fan-in & & $\left(A^{T} A^{2}\right)_{i i}$ & $\begin{array}{l}d_{i, i n}\left(d_{i, i n}-1\right) \\
=\sum_{j \neq k} a_{j i} a_{k i}\end{array}$ & $\left(W^{\left[\frac{2}{3}\right] T}\left(W^{\left[\frac{2}{3}\right]}\right)^{2}\right)_{i i}$ & $\left(s_{i, i n}^{\left[\frac{1}{2}\right]}\right)^{2}-s_{i, i n}$ \\
\hline Fan-out & & $\left(A^{2} A^{T}\right)_{i i}$ & $\begin{array}{l}d_{i, \text { out }}\left(d_{i, \text { out }}-1\right) \\
=\sum_{j \neq k} a_{i j} a_{i k}\end{array}$ & $\left(\left(W^{\left[\frac{2}{3}\right]}\right)^{2} W^{\left[\frac{2}{3}\right] T}\right)_{i i}$ & $\left(s_{i, \text { out }}^{\left[\frac{1}{2}\right]}\right)^{2}-s_{i, \text { out }}$ \\
\hline
\end{tabular}

possible triangle intensity if all weights connecting adjacent nodes were equal to 1 .

The proposed continuous definition fulfills all the conditions we put forward above, and to the best of our knowledge it is the only one to do so. Similarly to previous definitions, the proposed clustering coefficient can also be rewritten in terms of node properties:

$$
C_{i}=\frac{\left(W^{\left[\frac{2}{3}\right]}\right)_{i i}^{3}}{\left(s_{i}^{\left[\frac{1}{2}\right]}\right)^{2}-s_{i}}
$$

where $s_{i}=\sum_{j \neq i} w_{i j}$ is the normalized strength of node $i$ and $W^{[\alpha]}=w_{i j}^{\alpha}$ and $s_{i}^{[\alpha]}=\sum_{j \neq i} w_{i j}^{\alpha}$ are the fractional weight matrix and strength for any $\alpha \in \mathbb{R}$.

As for the previous definitions, the continuous clustering can be interpreted as a function of intensities and the binary clustering:

$$
C_{i}=\frac{n_{\Delta} \overline{I_{\Delta i j k}^{2}}}{n_{T} \overline{I_{T i j k}}}=\frac{\overline{I_{\Delta i j k}^{2}}}{\overline{I_{T i j k}}} C_{i}^{\text {bin }}=\frac{\operatorname{Var}\left(I_{\Delta i j k}\right)+{\overline{I_{\Delta i j k}}}^{2}}{\overline{I_{T i j k}}} C_{i}^{\text {bin }},
$$

with means $\overline{I_{\Delta i j k}}$ and $\overline{I_{T i j k}}$ and variance taken over all the triangles or triplets the node $i$ participates in, respectively. In the limit where all triangles associated with node $i$ have similar intensities, we can neglect the variance term, leaving $\frac{{\overline{I_{\Delta i j k}}}^{2}}{\bar{I}_{T i j k}} C_{i}^{\text {bin }}$. In this case, in contrast to the Zhang-Horvath definition [Eq. (5)], the absolute value of the intensity matters, not only its ratio to the maximum possible intensity. For a given average triangle intensity, the positive contribution of the variance implies that nodes with more variable intensities, i.e., at least one triangle with a high intensity, will have higher clustering coefficients than nodes with identical triangles of average intensity.

Finally, the global clustering can also be defined in a straightforward fashion. For simplicity, we define $\mathcal{I}_{\Delta, i}=$ $\sum_{j \neq k} I_{\Delta i j k}^{2}$ and $\mathcal{I}_{T, i}=\sum_{j \neq k} I_{T i j k}$, leading to $C_{i}=\mathcal{I}_{\Delta, i} / \mathcal{I}_{T, i}$. Using this definition, the continuous global clustering is ob- tained via the formula

$$
C_{g}=\frac{\sum_{i} \mathcal{I}_{\Delta, i}}{\sum_{i} \mathcal{I}_{T, i}}
$$

\section{Directed weighted clustering}

Fagiolo [19] proposed how to generalize clustering to directed networks. He defined the different patterns or motifs (shown in Table II) that can exist in these networks and adapted the Onnela definition [11] as the first weighted directed clustering coefficient. The Barrat definition [10] was generalized to directed graphs in Ref. [20] following the same distinction into Fagiolo's cycle, middleman, fan-in, and fanout motifs.

Similarly, the Zhang-Horvath definition [12] and the continuous definition can be generalized in a straightforward manner for directed networks. For this, we only need to redefine the intensities of each directed triangle and triplet motif (as shown in Table II for the continuous definition and in Table VII for the Zhang-Horvath definition). This simply requires replacing $A$ with $W$ in all expressions of $n_{\Delta, i}^{(m)}$ and $a$ with $w$ in all expressions of $n_{T, i}^{(m)}$. As the total directed clustering is defined as the sum of all modes, we can write it as

$$
C_{i}^{Z(\text { tot })}=\frac{I_{\Delta, i}^{Z(\text { tot })}}{I_{T, i}^{Z(\mathrm{tot})}}=\frac{\left(W+W^{T}\right)_{i i}^{3}}{\sum_{j \neq k}\left(w_{i j}+w_{j i}\right)\left(w_{i k}+w_{k i}\right)} .
$$

Finally, the continuous clustering can be extended for each directed mode (see Table II), and for the total directed clustering, this leads to

$$
C_{i}^{(\mathrm{tot})}=\frac{I_{\Delta, i}^{(\mathrm{tot})}}{I_{T, i}^{\text {(tot })}}=\frac{\frac{1}{2}\left(W^{\left[\frac{2}{3}\right]}+W^{\left[\frac{2}{3}\right] T}\right)_{i i}^{3}}{\left(s_{i, \text { tot }}^{\left[\frac{1}{2}\right]}\right)^{2}-s_{i, \text { tot }}-2 s_{i}^{\leftrightarrow}},
$$

with $s_{i, \text { tot }}=\sum_{j}\left(w_{i j}+w_{j i}\right)$ being the total strength, $s_{i, \text { tot }}^{\left[\frac{1}{2}\right]}=$ $\sum_{j}\left(w_{i j}^{\frac{1}{2}}+w_{j i}^{\frac{1}{2}}\right)$ being the total root strength, and $s_{i}^{\leftrightarrow}=$ $\sum_{j} \sqrt{w_{i j} w_{j i}}$ being the reciprocal strength. 
(a)

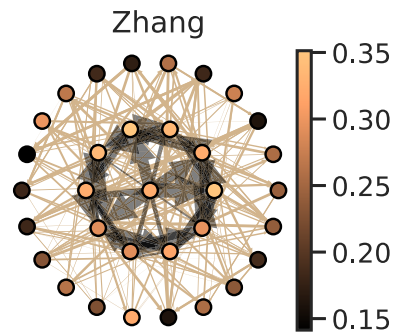

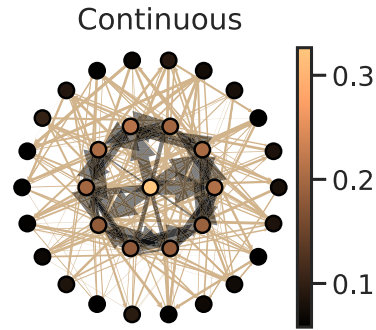
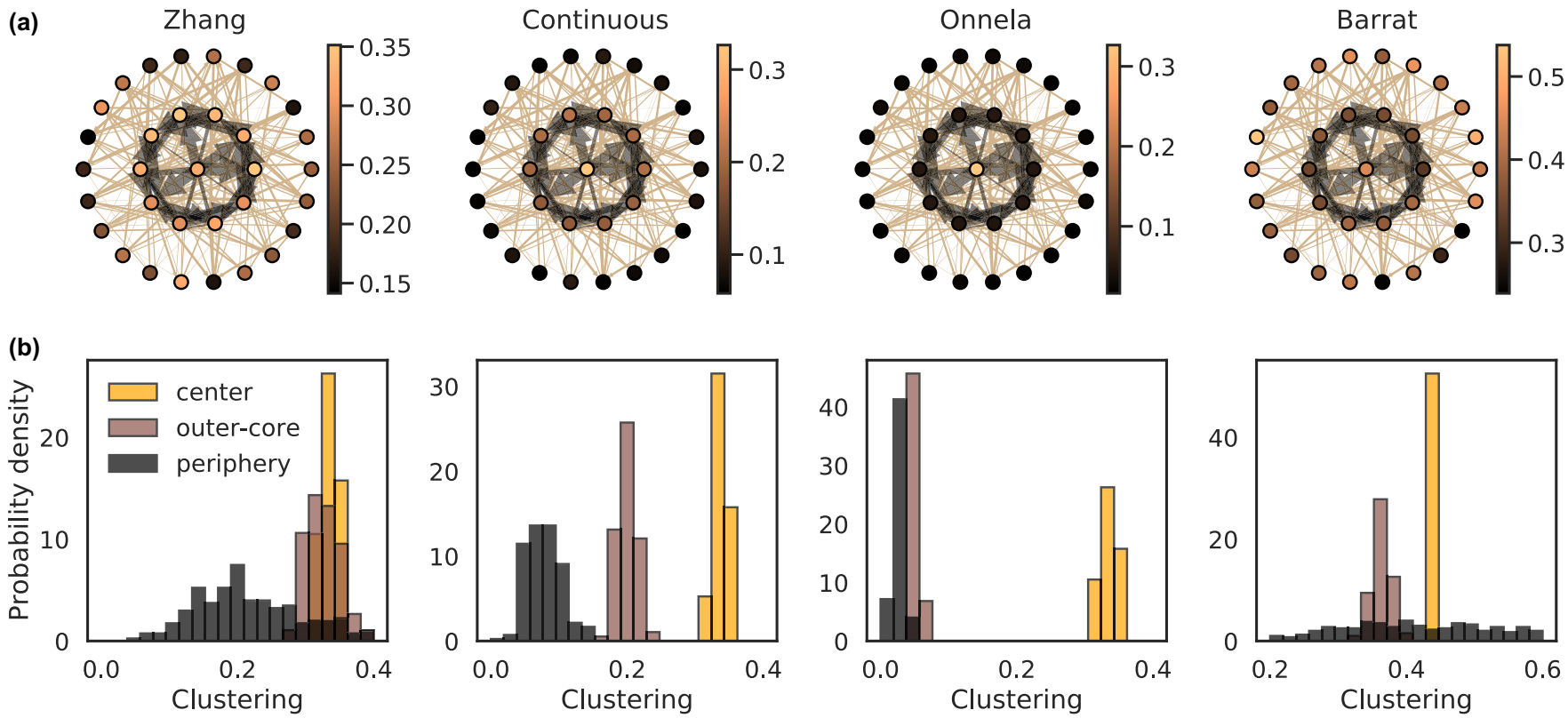

FIG. 1. Only the continuous clustering coefficient uncovers the true structure in the weighted core-periphery network. A network has 11 strongly connected core nodes (black edges) that interact with well-clustered periphery nodes with weaker connection strengths (light-brown edges); see Appendix E 1 for details on the network. (a) Graphical view of the network; the edge width gives the strength of the connection, and the node color gives its clustering coefficient. (b) Distribution of clustering coefficients for the three types of nodes over ten realizations of such a core-periphery network. Only the continuous definition differentiates between the central, the outer-core, and the periphery nodes. In all the other methods, the clustering coefficients of the 10 "outer-core" and 22 periphery nodes overlap: The Onnela definition only distinguishes the central node, whereas the Barrat and Zhang-Horvath definitions do not hint at a core-periphery structure.

As for the undirected case, the global clustering coefficient associated with each directed pattern can be obtained via the formula $C_{g}^{(m)}=\sum_{i} I_{\Delta, i}^{(m)} / \sum_{i} I_{T, i}^{(m)}$.

\section{THE ADVANTAGES OF FULLY WEIGHTED DEFINITIONS}

In this section we discuss the sensitivity to the weightencoded topological features and stability to noise in network measurements of the different clustering methods. A previous study [14] already noted the fact that previous definitions did not fulfill the continuity condition by analyzing the behavior of the different coefficients for nodes that participate in a single triangle. Table I illustrates some of these cases and shows that the proposed continuous definition is the only one to behave as expected.

Yet we note that the Zhang-Horvath definition is also very resilient to noise because, except for the corner cases associated with single triangles, its behavior is continuous in all other situations. Moreover, contrary to what was asserted in Ref. [14], it provides a perfectly sensible behavior given its interpretation of clustering as the ratio of the triangle intensity $I_{\Delta i j k}^{Z}=w_{i j} w_{i k} w_{j k}$ to its maximum possible intensity given the weights of node $i$ : $I_{\Delta i j k}^{Z(\max )}=I_{T i j k}^{Z}=w_{i j} w_{i k}$ if $w_{j k}=1$.

Because the Barrat and Onnela definitions [10,11] are the most well known and (to the best of our knowledge) the only methods implemented in popular graph libraries, we restrict our comparison to the Zhang-Horvath definition and these two definitions. A more comprehensive discussion of other definitions of the weighted clustering coefficient can be found in Appendix A.

\section{A. Sensitivity to weight-encoded topological features}

Here, we investigate how weighted structures can be detected or missed using different clustering definitions. As an example we consider weighted core-periphery graphs in Fig. 1. There, core nodes are characterized by both a dense binary connectivity and large weights, whereas periphery nodes display both sparser connectivity (though they still have large degrees) and weaker weights; for more details, see Appendix E 1. We generate ten realizations of the network and consider distribution of clustering coefficients of different types of nodes. Continuous definition leads to distinct clustering of different types of nodes, making the true structure of the network clearly visible already in the clustering distributions. Because of their hybrid nature, the Barrat and Onnela definitions cannot capture this underlying structure as it is mostly encoded in the weights and not at all in the degrees (core nodes are not binary hubs). Though it is purely weighted, the Zhang-Horvath definition is also not suited to detect this type of weighted structure because its interpretation of a node's clustering only accounts for the relative triangle intensity given the node's weights.

The continuous clustering is sensitive to any topological property that is encoded via specific weight distributions. Furthermore, in contrast to the Zhang-Horvath definition, it accounts not only for the ratio of the triangle intensity over the triplet intensity (how strong the triangles are compared with the maximum possible value given the node's weights) but also for the absolute value of the intensity: A weak triangle, even if it corresponds to the highest possible value given the node's weights, will decrease the node's clustering 
(a)

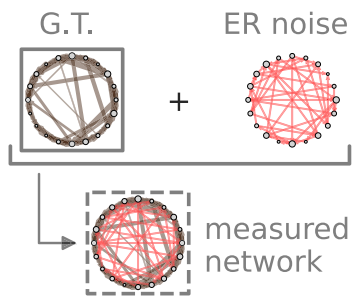

(e)

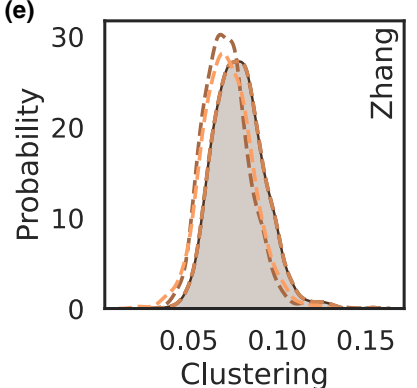

(b)

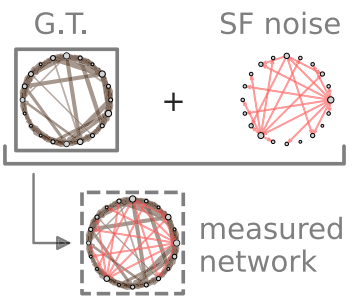

(f)

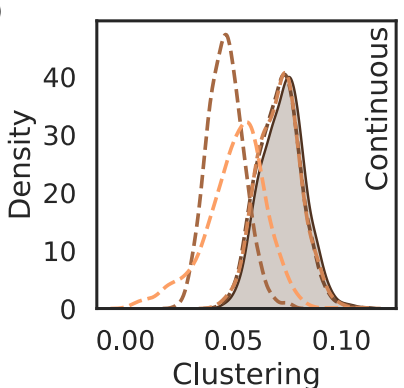

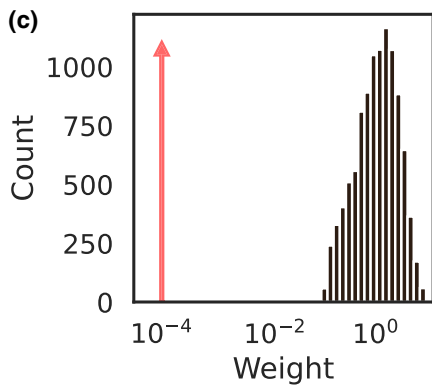

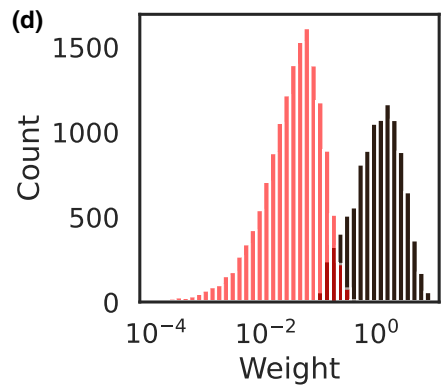

(g)

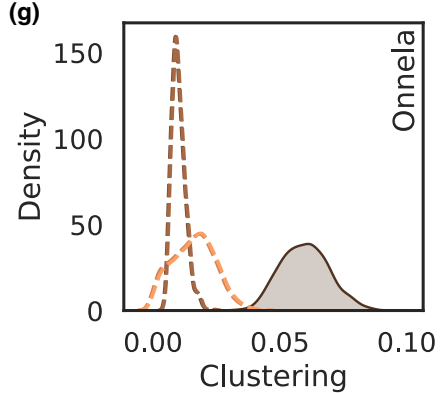

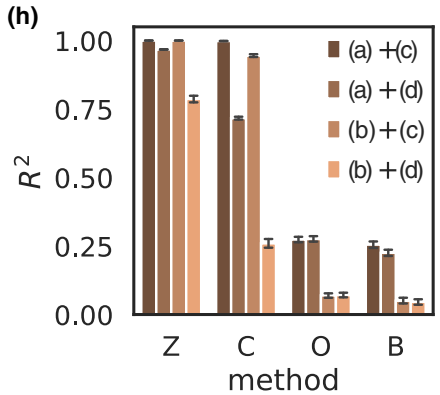

FIG. 2. Fully weighted methods are less sensitive to spurious edges. A "measured network" can be represented as the union of a "ground truth" (G.T.) — here, a Watts-Strogatz network in dark brown—and spurious small-weight connections ("noise" graphs with (a) random [ErdôsRényi (ER)] or (b) scale-free (SF) connectivity, in red). We assess the influence of the weight distribution of spurious connections (red) by checking weights that are (c) all equal and small or (d) following an exponential distribution and overlapping with the real weights (dark brown). (e)-(g) Ground-truth clustering distribution (filled dark brown) compared with the distributions associated with the measured networks for each method (dashed lines). Weight and noise types, combining noise shown in (a) or (b) with weight shown in (c) or (d), which we refer to as conditions "(a)+(c)," "(a)+(d)," "(b)+(c)," and "(b)+(d)," are associated with colors from brown to orange in the same order as in (h). Distributions associated with the exponential noise [conditions (a)+(d) and (b)+(d)] differ most from the original distributions for the Zhang-Horvath and continuous clusterings [(e) and (f)] and are broader for the Onnela clustering (g). (h) Correlation between the ground-truth clustering and clustering in measured networks for indicated spurious edge topology and weights. Fully weighted clusterings retain most of the correlation for condition (a)+(d), with $R^{2}>0.55$, and only lose the original information for condition (b) $+(\mathrm{d})$. The results were obtained for ten realizations of the spurious edges; error bars give confidence intervals. Network properties are detailed in Appendix E. Z, C, O, and B, Zhang-Horvath, continuous, Onnela, and Barrat definitions, respectively.

in the continuous definition whereas it increases it with the Zhang-Horvath method. In that sense, the continuous clustering provides a more global evaluation of the clustering coefficient compared with the Zhang-Horvath definition that provides more local information.

The Barrat definition has several limitations because it is close to being weight insensitive $[11,21]$. It is particularly unsuitable for assessing networks with a potentially large number of low-weight spurious connections or very heterogeneous weight distributions. For this reason, we will mostly leave this clustering aside in the rest of this paper.

\section{B. Continuity and resilience to noise}

The stability of a network measure to noise is of particular importance for networks that are obtained via experimental measurements since these are often subject to noise and statistical biases, notably for inferred networks. Methods abiding by the continuity condition are especially resilient to the presence of low-weight spurious edges. Violation of continuity can have significant and pervasive consequences for inference of network properties in many network structures. We have already seen the simple examples in Table I; here, we demonstrate that they are not just corner cases, but occur in larger, real-world networks.
We illustrate the impact of spurious edges on measured clustering coefficients using the example of Watts-Strogatz small-world networks. We consider different topologies for the subnetwork formed by the spurious edges: either an Erdős-Rényi random network [Fig. 2(a)], associated with uncorrelated noise, or a scale-free network [Fig. 2(b)], which would correlate noise with certain nodes in the network. Additionally, weights on the spurious edges could be much smaller than the weight of the actual edges [Fig. 2(c)] or have an overlapping distribution [Fig. 2(d)]. We refer to the combinations of these noise and weight types as conditions "(a)+(c)," "(a)+(d)," "(b)+(c)," and "(b)+(d)." Both fully weighted methods are unaffected by low noise [conditions (a) + (c) and (b)+(c)] and are also less influenced by the spurious edges when their weights are large enough to overlap with the real weight distribution [conditions (a)+(d) and (b)+(d)]. On the other hand, because hybrid methods explicitly depend on the nodes' degrees, they are very susceptible to the presence of spurious edges [Figs. 2(g) and 2(h)].

The difference in behavior between the methods can be easily explained by a first-order expansion. We consider change in the clustering coefficient of a node $i$ with degree $d_{i}$ after addition of a spurious edge $e=(i, v)$ with weight $\epsilon \ll 1$. For the Barrat and Onnela methods, the new clustering 
coefficient becomes

$$
\begin{aligned}
C_{i}^{O^{\prime}} & =\frac{I_{\Delta, i}^{C}+O\left(\epsilon^{\frac{1}{3}}\right)}{d_{i}\left(d_{i}+1\right)}=\frac{d_{i}-1}{d_{i}+1} C_{i}^{O}+O\left(\epsilon^{\frac{1}{3}}\right) \stackrel{\epsilon \rightarrow 0}{\rightarrow} C_{i}^{O}, \\
C_{i}^{B^{\prime}} & =\frac{I_{\Delta, i}^{B}+\sum_{k} \frac{\epsilon+w_{i k}}{2} a_{v k} a_{i k}}{I_{T, i}^{B}+s_{i}+\epsilon}=\frac{d_{i}-1}{d_{i}} C_{i}^{B}+O(\epsilon) \stackrel{\epsilon \rightarrow 0}{\rightarrow} C_{i}^{B},
\end{aligned}
$$

meaning that, for both methods, the coefficients will deviate from the original clustering $C_{i}^{O / B}$ by a noninfinitesimal value, even when the perturbation was infinitesimal; see Appendix $\mathrm{C}$ for a complete derivation.

On the other hand, the continuous clustering becomes

$$
\begin{aligned}
C_{i} & =\frac{I_{\Delta, i}+\sum_{k \sim i}\left(\epsilon w_{v k} w_{k i}\right)^{\frac{2}{3}}}{I_{T, i}+2 s_{i}^{\left[\frac{1}{2}\right]} \sqrt{\epsilon}} \\
& =C_{i}\left(1-\frac{2 s_{i}^{\left[\frac{1}{2}\right]} \sqrt{\epsilon}}{I_{T, i}}+O\left(\epsilon^{\frac{2}{3}}\right)\right) \\
& =C_{i}+O(\sqrt{\epsilon}) \underset{\epsilon \rightarrow 0^{+}}{\longrightarrow} C_{i},
\end{aligned}
$$

showing only an infinitesimal deviation to the similarly infinitesimal perturbation.

Similarly, except for the single-triangle cases discussed in Table I, the Zhang-Horvath clustering becomes

$$
C_{i}^{Z^{\prime}}=\frac{I_{\Delta, i}^{Z}+O(\epsilon)}{I_{T, i}^{Z}+O(\epsilon)}=C_{i}^{Z}+O(\epsilon) \underset{\epsilon \rightarrow 0^{+}}{\longrightarrow} C_{i}^{Z} .
$$

It is worth noting that one continuity issue, associated with nodes participating in only one triangle, occurs for all definitions but the continuous one. Since this situation is pervasive in networks with low degree or binary clustering, using the continuous clustering definition can be of particular importance in such cases; see Appendix F 4.

\section{APPLICATION TO REAL-WORLD NETWORKS}

\section{A. Mouse mesoscale connectome}

In neuroscience, the networks on different scales give a vital piece of information to understand the brain better. Unsurprisingly, connectomics, the mapping of the connections in the nervous system, has gained significant attention and developed dramatically over the last years. Most of the networks in neuroscience are weighted, and the obtained connectivities are either measured or inferred, making them a typical example for the challenges discussed above. The mouse mesoscale connectome [22] is a fascinating example of such networks, both because it provides information about the entire mouse brain and because it contains an evaluation of the probability of false positives for all connections.

Here, we investigate how the choice of the clustering coefficient definition can alter the results. The network is very inhomogeneous, with broadly varying degrees [Fig. 3(a)]. The edges in the mesoscale connectome are assigned $p$ values that quantify their probability to correspond to a real physical connection ( $p$ denotes the probability of the connection to be a spurious edge). They have therefore different significance levels, with only $13 \%$ of all edges having $p$ values smaller than 0.01 , i.e., only $13 \%$ of all edges have a probability to be spurious that is lower than $1 \%$. We consider a thresholding procedure, where at each level of the threshold $\left(p_{\max }\right)$, only the edges with smaller $p$ values are kept. This procedure can be seen as an attempt to remove spurious edges, though no correct threshold level is known. Compared with the hybrid method, both fully weighted definitions are much less sensitive to thresholding [Figs. 3(b) and 3(c)]. Thus we see that the resilience to noise we showed analytically and on toy networks is relevant for a real-world network. Furthermore, the resilience is not limited to the general shape of the distribution but indeed preserves the precise values and ranks: A larger fraction of the nodes displaying high clustering in the full graph are still among the highest ranking nodes in the thresholded graphs when fully weighted methods are used compared with the hybrid method [Figure 3(e)].

The clustering coefficient is a network measure that captures features beyond purely local parameters, such as degree or strength. However, as we see for the mouse connectome, the hybrid method strongly correlates with the average weight associated with a node $i: s_{i} / d_{i}$. At the same time, the fully weighted definitions are much less correlated with it [Fig. 3(d)]. As a result, they can bring more independent information regarding the weighted network structure than the hybrid method. This trend is even stronger for the ZhangHorvath definition, which does not account for the absolute intensity of triangles. In contrast, the continuous definition provides some intermediate behavior as the intensity of triangles often correlates, if only in part, with the average weight associated with the node.

Finally, though the continuous and Zhang-Horvath definitions often provide somewhat similar results, they may differ significantly, e.g., for the cerebellar cortex in Fig. 3(f). Combining the results of both methods can thus be informative, for example, to single out nodes that possess only weak connections (and will therefore register as weakly clustered for the continuous definition) yet connect to other nodes that are strongly connected (thus registering as strongly clustered for the Zhang-Horvath definition).

\section{B. Decentralized social media: The Fediverse}

The Fediverse [23] is a set of federated social media that can communicate via a collection of common protocols [24], the most well known being ActivityPub. This network can be seen as a set of alternatives to corporate platforms such as Facebook or Twitter. Social media on the Fediverse usually promote ideas of decentralization, interoperability, free/libre and open-source software (FLOSS), and the absence of algorithmic filters in favor of human curation and moderation.

We analyze here a snapshot of this network that was obtained in 2018 by Zignani et al. [25] (data are available [26]). In contrast to the original publication, we chose here to look at the mesoscale level, i.e., at connections between instances (the equivalent of a community on the Fediverse, where at least one, but up to several thousand users can have an account). This mesoscale view leads to a network of weighted directed interactions between communities of strongly connected users. Indeed, users of a single instance (the technical name for a community on the Fediverse) can see and interact with all public messages posted by other members on that same server. At the same time, they can only see a subset of 
(a)

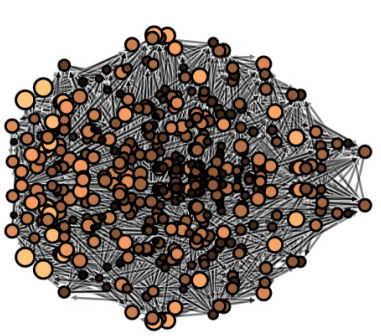

(c)

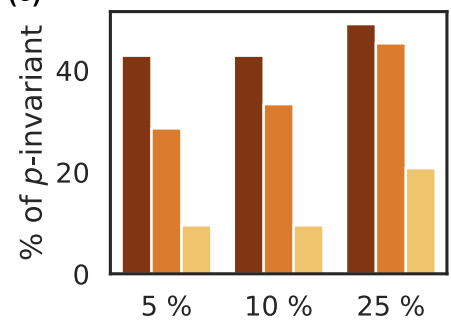

Fraction of top-clustered nodes

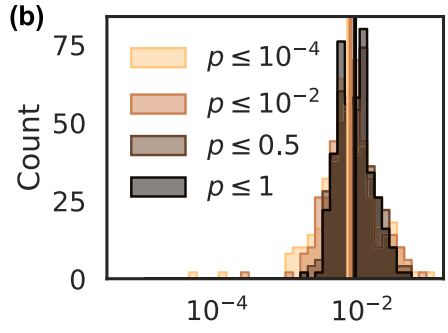

(d)

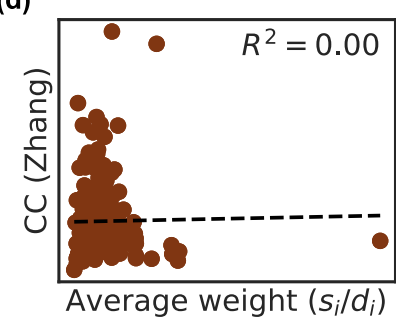

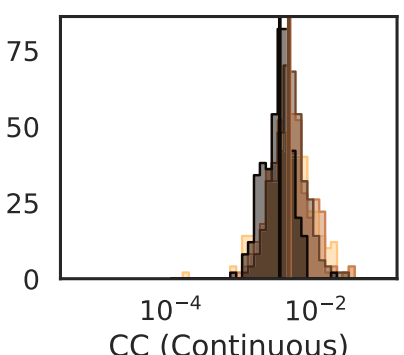

CC (Continuous)

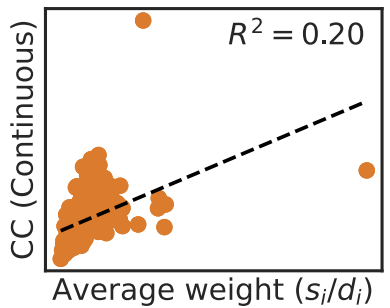

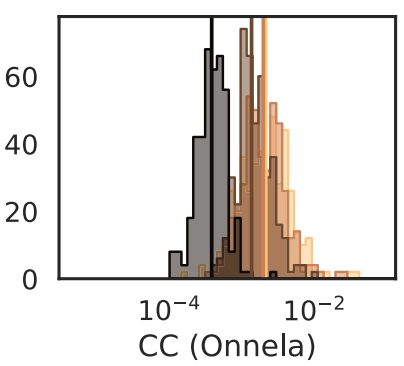

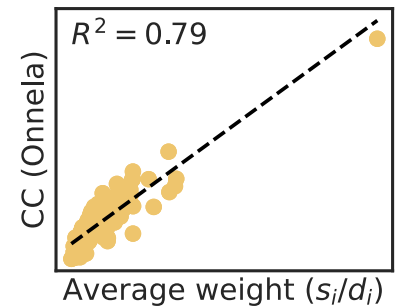

(e)

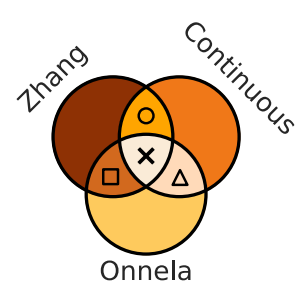

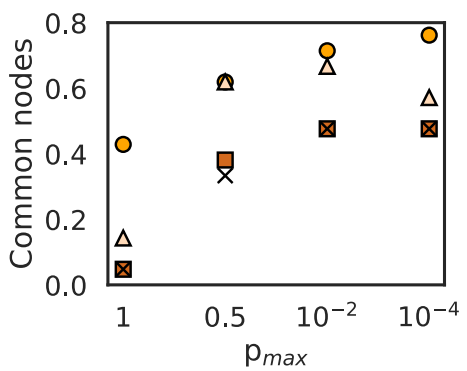

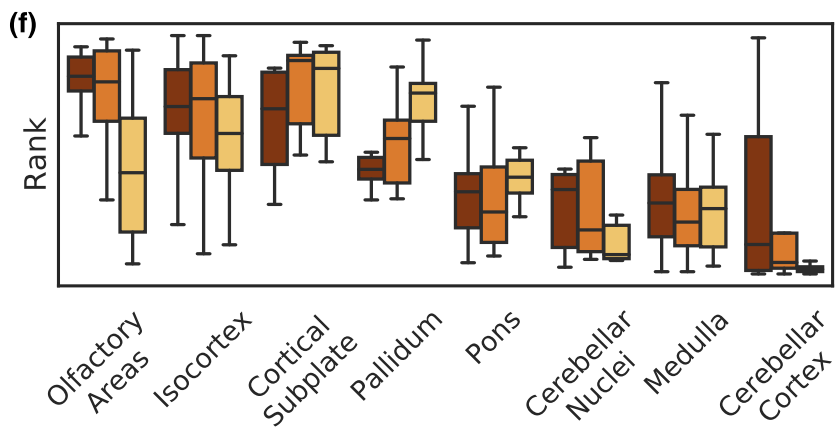

FIG. 3. For the mouse connectome, different clustering methods give significantly different results. (a) Top view of the mouse brain; node size indicates the total degree, and node color indicates the out-degree (lighter colors for higher degrees). (b) Distribution of the total clustering coefficients if only edges with the $p$ value $p<p_{\max }$ are preserved. There are smaller changes in clustering distribution for fully weighted definitions than for the Onnela definition. From pale yellow to dark gray, thresholding keeps 9, 13, 32, and 100\%, respectively, of the original network. (c) The fraction of the nodes with the highest total clustering (top 5, 10, and 25\%) that are preserved across all subsamplings in (b) for the Zhang-Horvath (brown), continuous (orange), and Onnela definitions (pale yellow). (d) Correlation of the three total clustering definitions with average total node weight $\left(s_{i} / d_{i}\right)$ shows that fully weighted definitions capture additional information beyond degree and strength. (e) Fraction of the $10 \%$ highest clustering nodes that are common between two of the definitions (filled markers in the right panel include the central region of the left panel) or among all three definitions (black crosses, central region) as shown in the Venn diagram. (f) Clustering ranks of the areas within brain regions (showing which regions contain nodes with high clustering coefficients) can significantly vary depending on the definition (Zhang-Horvath, brown; continuous, orange; and Onnela, pale yellow).

the posts from people on other servers (either because they follow their author or because other members of the instance follow the author or shared this specific post).

For each instance $I_{1}$, an edge towards another instance $I_{2}$ means that at least one user on $I_{1}$ follows at least one member of $I_{2}$. The precise value of the weight associated with this edge gives the fraction of all followers from the source instance that are associated with members of the target instance. The weights are thus a proxy to characterize the fraction of the community's attention that is associated with content produced by another community; this means of course that, for each node, its outgoing strength $s_{o}$ is equal to 1 . Note that in this network, information flow occurs in the direction opposite to that of the edge, because the directed edge denotes that the source is paying attention to what the target posts.

The network snapshot contains 3825 nodes corresponding mostly to instances running Mastodon [27], one of the most prominent microblogging platforms on the Fediverse. These
3825 nodes represent more than half of the entire network and are connected via 81371 edges. A chord diagram of connections between locations shows that, besides the fact that the position of most instances is unspecified (UNS), the largest hosting countries are Japan, the United States, and France, with Japanese and French communities interacting mostly among themselves [Figs. 4(a) and 4(b)]. The network is both very sparse and strongly heterogeneous, with a median degree of 5 but node degrees and sizes varying over 3-5 orders of magnitude. This broad distribution has notable implications for different clustering definitions. For the Zhang-Horvath definition, it increases the likelihood of running into the corner cases of single triangles, increasing the average clustering compared with the other methods. For the Onnela definition, it strongly correlates clustering values to the degree (and thus to the size) of the instance.

As for the mouse network, all weighted methods lead to results that differ strongly from the binary clustering [Figs. 4(d) 
(a)
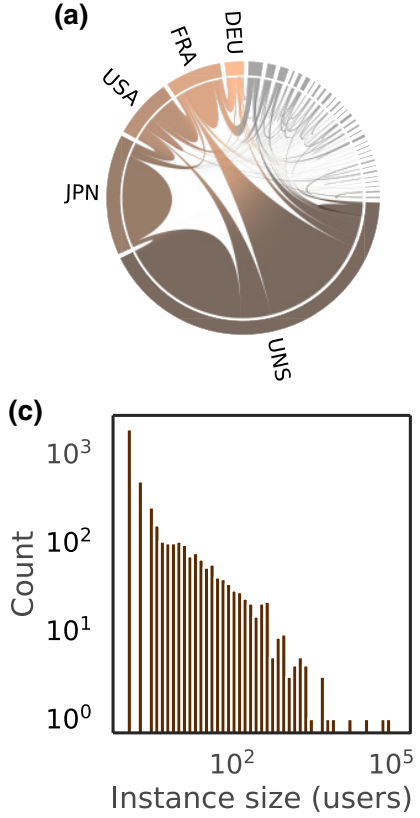

(b) (d)

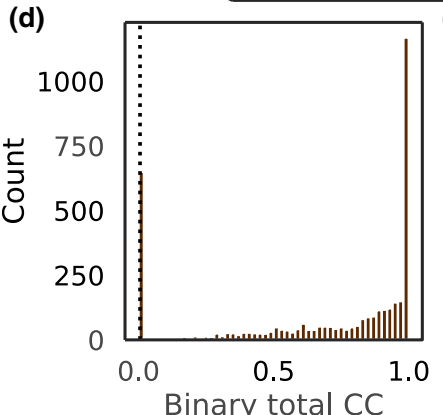

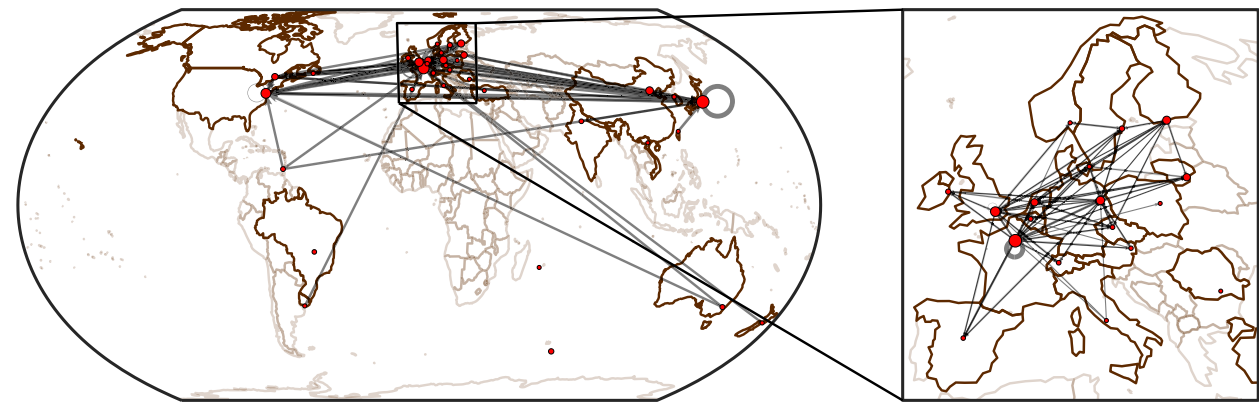

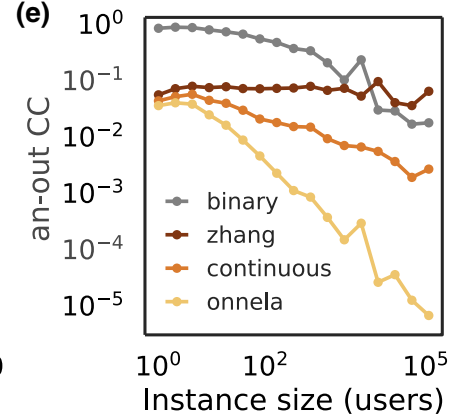

(f)

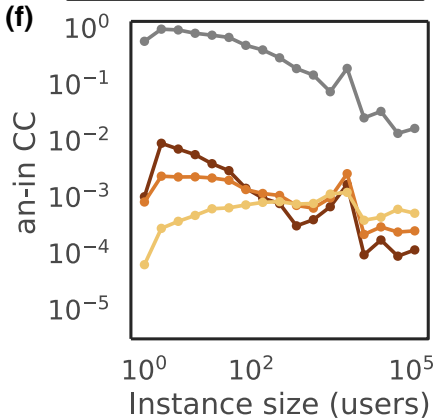

FIG. 4. Properties of the network of Fediverse instances. (a) Chord diagram of connections between locations (DEU, Germany; FRA, France; JPN, Japan; UNS, unspecified; USA, United States of America). (b) Spatial representation of the network showing connections that amount to at least $1 \%$ of the strongest connection; nodes are placed on each country's capital, and their sizes represent the number of instances hosted in that country. The zoom on Europe shows all connections in the European subnetwork. (c) The count of users per instance follows a heavy-tailed distribution. (d) The network displays strong structural clustering, most nodes with nonzero in- and out-degrees displaying binary clustering values close to 1, whereas the expected value for an Erdős-Rényi network with the same number of edges would be almost zero (dotted line). (e) The median values of the fan-out clustering for different instance sizes show that the strong heterogeneity of the network can have a notable influence for the Onnela method (yellow), whereas the Zhang-Horvath (dark brown) and continuous methods (orange) display much weaker correlation with the size of the instance. (f) The fan-in motifs have intensities that are several orders of magnitude lower than fan out and display weaker dependency on the instance size. The binary (gray) clustering coefficient misses the difference between fan out and fan in, which predominantly relies on the weights' effect.

and $4(\mathrm{e})]$. Some of the results from the hybrid method tend to correlate strongly with some first-order properties of the nodes [Fig. 4(e)], whereas the fully weighted methods bring more independent information. The Fediverse network displays a peculiar feature as its fan-out and fan-in clusterings differ significantly despite the usual correlation between these two patterns, as can be seen from the comparison of Figs. 4(e) and 4(f).

\section{Using local clustering to infer dynamical properties}

Analysis of the clustering coefficient for different structured patterns offers a way to obtain a precise idea of the critical dynamical patterns within a network. To determine the significance of a particular pattern, we compare its prominence in the original network with its prominence in null-model networks obtained via appropriate randomization. For the mouse brain, as a randomized control, we take the original network and only shuffle the weights, thus preserving the weight distribution and binary structure. Comparing the actual values in the original graphs with those of the randomized graphs, we can see the importance of looking at fully weighted measures.

Both the Zhang-Horvath and continuous definitions identify the preference of the mouse brain network for redundant information flows, whereas the hybrid method of Onnela does not capture this feature. Redundant information transfer in the brain is indeed associated with situations where a signal can be transferred not only directly from one node to another, but also indirectly via a third (middleman) node, as can be seen in middleman, fan-in, and fan-out patterns (cf. Table II). This overexpression of redundant patterns is visible, for the fully weighted methods, in the high values of the middleman, fan-in, and fan-out motifs in Fig. 5(a).

The situation for the Fediverse is significantly more complex. Indeed, $98 \%$ of all edges belong to at least one triangle, and some are involved in up to thousands of triangles. A deeper understanding of the clustering requires precise investigation of how the weight distribution correlates with specific patterns of triangles. For instance, though fan-in and fan-out patterns co-occur and are thus usually correlated, the Fediverse displays an unexpected discrepancy between the weights associated with the two patterns, as seen in Fig. 5(b), which notably differentiates it from the mouse connectome (see also Appendix F 3).

\section{DISCUSSION}

In this paper, we introduced directed weighted definitions for clustering analysis. Using analytic derivations, generated network models, and real data, we showed that the behavior of fully weighted measures displayed enhanced sensitivity, selectivity, and robustness compared with hybrid measures. To facilitate access to these measures, all clustering methods 

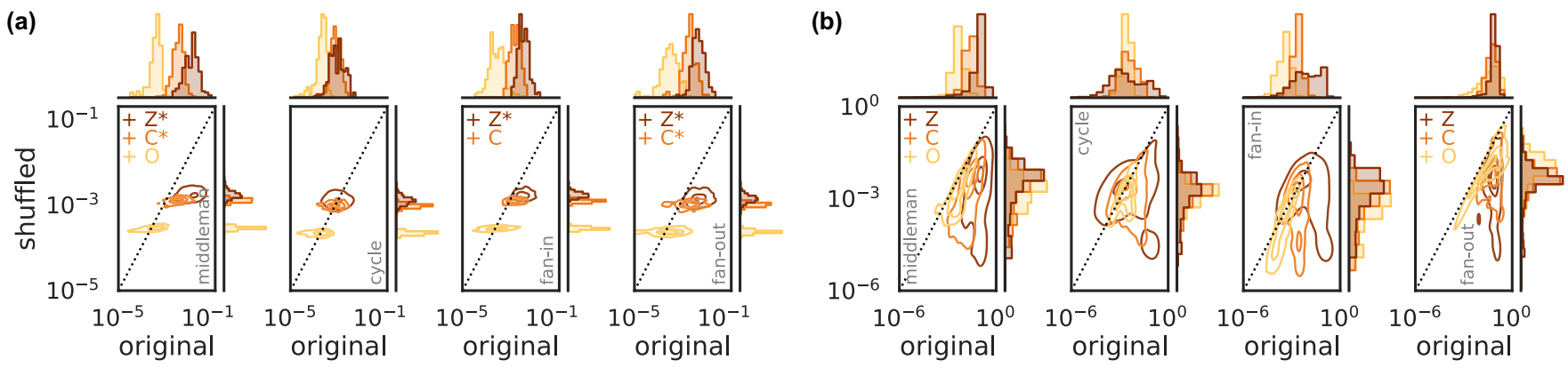

FIG. 5. Different structures of clustering patterns and information flow in the mouse brain and the Fediverse. The original clustering values are compared with graphs with the same adjacency matrix but shuffled edge weights (mouse, 10 uniformly shuffled networks; Fediverse, 200 graphs shuffled across all outgoing edges of each node to preserve out-strength normalization). The contours show different density levels of the point clouds associated with the original and shuffled pairs of clustering coefficients. (a) The Zhang-Horvath and continuous definitions capture the redundancy of information flow in the mouse brain (the middleman, fan-in, and fan-out motifs are higher in the original graph). However, the Onnela method does not capture this feature. (b) For the Fediverse, only fan-out and middleman motifs are significantly stronger than in the random graphs. Patterns where the original values are significantly greater than the randomized ones are marked by a + and the initial of the method (brown Z, Zhang-Horvath method; orange C, continuous method; yellow O, Onnela method). The original clustering is considered to be significantly higher (lower) if $75 \%$ of the points are above (below) the dotted identity line. The addition of $*$ denotes one-sided fractions greater than $95 \%$.

were implemented in PYTHON and made compatible with the three main libraries in this language (NETWORKX, IGRAPH, and GRAPH-TOOL) [28].

The use of a specific weighted clustering coefficient should be an informed choice, based on the precise questions and objects of study and thus on the specific properties that a relevant measure should fulfill to answer these questions. We therefore wish to highlight the importance of continuity as a potentially crucial notion for networks with highly heterogeneous weight distribution or numerous spurious edges with low weights. In previous studies, slight variations on this notion had been introduced mathematically [15] or hinted at by the study of corner cases [14]. However, the continuity property was inadvertently considered only in particular classes of networks in Ref. [15] (disregarding, for example, small-degree cases), erroneously marking previous methods as continuous. The other study (Ref. [14]) asserted discontinuity of previous measures simply as a feature, without discussing its implications or ways to avoid it. Our proposal of fully continuous clustering methods based on simple mathematical principles and requirements fully solves the issue of continuity. In addition, we show in Appendix D that these principles can be extended to other measures such as the local closure.

We discussed how each weighted definition is associated with a specific interpretation of weighted clustering as a function of the binary clustering, triangle, and triplet intensities (see Appendix B and Table VI for a summary). Correspondingly, these interpretations are associated with specific properties of each clustering coefficient. In rare cases where, by design, there are edges with small weights that should be treated significantly differently from an absence of connection, researchers may want to check whether the properties of the Barrat or Onnela definitions fit their needs or if they should come up with new, more appropriate definitions. In many other cases, combining mathematical analysis and concrete examples, we asserted that fully weighted measures outperform hybrid ones to evaluate clustering in networks with large numbers of spurious edges with small weights. We expanded the results from previous studies comparing existing weighted definitions [14-16,21], extending their definition to directed networks and providing complete mathematical justifications for previous observations regarding linearity and continuity.

Our analyses show that either of the fully weighted definitions may be preferred depending on the network properties. For networks with large degrees, the Zhang-Horvath definition may be preferred if the number of low-weight spurious edges is very high, as it is least susceptible to noise. In networks with heterogeneous weight distributions where the absolute value of triangle strength is of interest, we showed that the continuous definition provides more relevant results than the Zhang-Horvath definition. Indeed, in networks involving fluxes of goods or matter as well as for information processing (e.g., in brain or telecommunications networks), one may be interested in the absolute amount that can be transferred between nodes, making nodes with small weights of little relevance. A similar issue occurs for networks where many nodes participate in a single triangle (see Appendix F 4) making the Zhang-Horvath clustering noncontinuous. In such networks, the use of the Zhang-Horvath definition is likely to assign high clustering to single-triangle nodes with low weights, whereas the continuous definition will not, as was shown in Fig. 1.

Finally, we illustrate the usefulness of weighted clustering methods to investigate clustering in the examples of a connectome and a decentralized social network. The fully weighted methods were especially suitable to reveal key differences in their weighted structural properties. Indeed, we showed that middleman, fan-in, and fan-out patterns, characteristic of pathways enabling redundant information transfer between nodes, were overexpressed in the mouse brain. In the Fediverse, the methods revealed an unexpected discrepancy between fan-in and fan-out modes, probably associated with social interaction patterns that would mandate further investigation. 
TABLE III. Undirected weighted clustering coefficients of vertex $i$ (solid circles) for different weight configurations. Solid lines depict edges of weight $w=\max (w)=1$, whereas dotted lines are associated with edges with vanishing weight $\epsilon$. Only the proposed continuous clustering (bottom row) displays the required properties, compared with the Zhang-Horvath $\left(C^{Z}\right)$, Holme et al. [17] $\left(C^{H}\right)$, and Miyajima-Sakuragawa (hm) definitions $\left(C^{M, h m}\right)$.

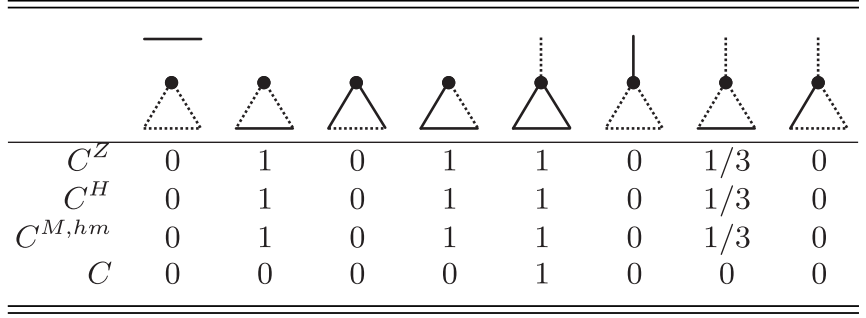

\section{ACKNOWLEDGMENTS}

This research was funded by a Humboldt Research Fellowship for Postdoctoral Researchers and a Sofja Kovalevskaja Award from the Alexander von Humboldt Foundation, endowed by the Federal Ministry of Education and Research. We thank the anonymous reviewers for their comments, which helped to significantly improve our manuscript. We acknowledge the support from the BMBF through the Tübingen AI Center (FKZ 01IS18039B).

\section{APPENDIX A: LIMITATIONS OF OTHER FULLY WEIGHTED DEFINITIONS}

See Table III for a complete comparison of the fully weighted clustering definitions.

\section{Holme et al. [17]}

Most studies consider the definition of Holme et al. [17] to be

$$
C_{i}^{H}=\frac{\sum_{j k} w_{i j} w_{i k} w_{j k}}{\max _{i j}\left(w_{i j}\right) \sum_{j k} w_{i j} w_{j k}},
$$

which would make it inconsistent with the binary definition. However, the discussion in their paper states that consistency was one of their requirements, letting us think that they actually meant to define it as

$$
C_{i}^{H}=\frac{\sum_{j \neq k} w_{i j} w_{i k} w_{j k}}{\max _{i j}\left(w_{i j}\right) \sum_{j \neq k} w_{i j} w_{j k}},
$$

which would make it equal to the Zhang-Horvath definition [12], and we will therefore not consider Eq. (A1) here.

\section{Miyajima and Sakuragawa [18]}

Miyajima and Sakuragawa [18] define a multitude of generalized clustering coefficients based on the use of an arbitrary function $h: \mathbb{R}^{2} \rightarrow \mathbb{R}$ such that

$$
C_{i}^{M, h}=\frac{\sum_{j k} h\left[h\left(w_{i j}, w_{i k}\right), w_{j k}\right]}{\sum_{j \neq k} h\left[h\left(w_{i j}, w_{j k}\right), \max _{l m}\left(w_{l m}\right)\right]} .
$$

More specifically, Wang et al. [15] argue in favor of the use of a specific version using the harmonic mean $(\mathrm{hm})$ :

$$
C_{i}^{M, h m}=\frac{\sum_{j k} \frac{2}{\frac{2}{\frac{1}{w_{i j}}+\frac{1}{w_{i k}}}+\frac{1}{w_{j k}}}}{\sum_{j \neq k} \frac{2}{\frac{1}{\frac{1}{w_{i j}}+\frac{1}{w_{i k}}}+\frac{1}{\max _{l m}\left(w_{l m}\right)}}},
$$

which we will refer to as the Miyajima-Sakuragawa (hm) definition. However, this definition suffers from three major shortcomings:

(i) Despite what is asserted by the authors, it does not fulfill the continuity condition.

(ii) It is not locally linear, meaning that two nodes that have the same neighborhood but with all weights differing by a factor $\lambda$ will not have the ratio of their clustering coefficients equal to $\lambda$.

(iii) It introduces an undesired asymmetry in the definition of the triangle intensity: For a given triangle $\Delta_{i j k}$, the computed intensity will be different for each node as it depends on which one is considered as $i$.

\section{APPENDIX B: COMPARISON OF CLUSTERING PROPERTIES}

Multiple properties of the main definitions for the clustering coefficient are listed in Table IV. These properties depend on the definitions of triangle and triplet intensity that are recapitulated in Table $\mathrm{V}$.

The different formulas for the intensities lead to different interpretations of weighted clustering (Table VI). The Barrat and Zhang-Horvath definitions only quantify ratios of triangle and triplet strength, while the Onnela and continuous definitions are sensitive to the absolute value of the triangle intensity. The latter provides an intermediate interpretation between the Zhang-Horvath and Onnela definitions as it reacts to both the ratio of intensities and the absolute value of the triangle intensity.

\section{APPENDIX C: DERIVATION OF THE EVOLUTION OF HYBRID CLUSTERING COEFFICIENTS}

\section{Barrat method}

Upon addition to a graph $G(N, E)$ of an edge $(i, v)$ of weight $\epsilon$, giving $G^{\prime}\left(N, E^{\prime}\right)=E+\{(i, v)\}$, one can compute the evolution of the initial clustering coefficient $C_{i}^{B}$ to its new value $C_{i}^{B^{\prime}}$ by rewriting the definition from Ref. [10] as in Ref. [14]:

$$
\begin{aligned}
C_{i}^{B} & =\frac{1}{d_{i}\left(d_{i}-1\right)} \sum_{j \neq k} \frac{w_{i j}+w_{i k}}{2 \overline{w_{i}}} a_{i j} a_{i k} a_{j k} \\
& =\frac{1}{d_{i}\left(d_{i}-1\right)} \sum_{j \neq k} \frac{w_{i j}}{\overline{w_{i}}} a_{i j} a_{i k} a_{j k} .
\end{aligned}
$$

From this, we can deduce the following relationship between the Barrat definition and the binary clustering coefficient $C_{i}^{\text {bin }}$ :

$$
C_{i}^{B}=\frac{n_{\Delta, i} \overline{w_{i}^{\Delta}} / \overline{w_{i}}}{d_{i}\left(d_{i}-1\right)}=C_{i}^{\mathrm{bin}} \frac{\overline{w_{i}^{\Delta}}}{\overline{w_{i}}},
$$


TABLE IV. Comparison of the different properties of the different clustering coefficients. The Zhang-Horvath and continuous definitions fulfill the maximum number of desirable properties. An "X" means that the method possesses this property.

\begin{tabular}{lccccc}
\hline \hline Property & Barrat & Onnela & Miyajima-Sakuragawa (hm) & Zhang-Horvath & Continuous \\
\hline Consistent with binary definition & $\mathrm{X}$ & $\mathrm{X}$ & $\mathrm{X}$ & $\mathrm{X}$ & $\mathrm{X}$ \\
Normalized $(C \in[0,1])$ & $\mathrm{X}$ & $\mathrm{X}$ & $\mathrm{X}$ & $\mathrm{X}$ & $\mathrm{X}$ \\
All weights participate in $I_{\Delta}$ & & $\mathrm{X}$ & $\mathrm{X}$ & $\mathrm{X}$ & $\mathrm{X}$ \\
All weights participate equally in $I_{\Delta}$ & & $\mathrm{X}$ & $\mathrm{X}$ & $\mathrm{X}$ \\
Linear against local scaling of the weights & & $\mathrm{X}$ & $\mathrm{X}$ & $\mathrm{X}$ \\
Sensitive to weight permutations & $\mathrm{X}$ & & $\mathrm{X}$ \\
For a triangle $\Delta=(i, j, k), I_{\Delta}=I_{T} f\left(w_{j k}\right)$ & & & & $\mathrm{X}$ \\
Continuous & & & & $\mathrm{X}$ \\
\hline \hline
\end{tabular}

with $n_{\Delta, i}$ being the number of triangles in which node $i$ participates and $\overline{w_{i}^{\Delta}}$ being the average weight associated with edges connected to $i$ and participating in a triangle.

Notice that this expression also explains why the Barrat definition is so close to the binary clustering: For networks where weights are either rather homogeneous or not strongly correlated to triangles, $\overline{w_{i}^{\Delta}}$ and $\overline{w_{i}}$ become very close as the number of triangles per node increases.

Using Eq. (C2), the new clustering can be defined as

$$
\begin{aligned}
C_{i}^{B^{\prime}} & =C_{i}^{\mathrm{bin}^{\prime}} \frac{\overline{w_{i}^{\Delta}}}{\overline{w_{i}^{\prime}}} \\
& =\frac{n_{\Delta, i}^{\prime}}{d_{i}\left(d_{i}+1\right)} \frac{\frac{n_{\Delta, i} \overline{w_{i}^{\Delta}}+\left(n_{\Delta, i}^{\prime}-n_{\Delta, i}\right) \epsilon}{n_{\Delta, i}^{\prime}}}{\frac{d_{i} \overline{w_{i}}+\epsilon}{d_{i}+1}} \\
& =\frac{n_{\Delta, i} \overline{w_{i}^{\Delta}}}{d_{i}^{2} \overline{w_{i}}}+O(\epsilon) \\
& =\frac{d_{i}-1}{k} C_{i}^{B}+O(\epsilon) .
\end{aligned}
$$

\section{Onnela method}

As for the other definitions, the clustering from Ref. [11] can be defined as a function of the binary clustering:

$$
C_{i}^{O}=\frac{n_{\Delta} \overline{I_{\Delta i j k}^{O}}}{d_{i}\left(d_{i}-1\right)}=C_{i}^{\mathrm{bin}} \overline{I_{\Delta i j k}^{O}}
$$

TABLE V. Comparison of the formula for triangle and triplet intensity among the different clustering definitions for undirected networks.

\begin{tabular}{lcc}
\hline \hline Definition & Triangle $\left(I_{\Delta i j k}\right)$ & Triplet $\left(I_{T i j k}\right)$ \\
\hline Barrat & $\frac{w_{i j}+w_{i k}}{2 \overline{w_{i}}} a_{i j} a_{i k} a_{j k}$ & $d_{i}\left(d_{i}-1\right)$ \\
Onnela & $\left(w_{i j} w_{i k} w_{j k}\right)^{1 / 3}$ & $d_{i}\left(d_{i}-1\right)$ \\
Miyajima-Sakuragawa (hm) & $\frac{2}{\frac{2}{\frac{1}{w_{i j}}+\frac{1}{w_{i k}}}+\frac{1}{w_{j k}}}$ & $\frac{2}{\frac{1}{w_{i j}}+\frac{1}{w_{i k}}}$ \\
Zhang-Horvath & $w_{i j} w_{i k} w_{j k}$ & $w_{i j} w_{i k}$ \\
Continuous & $\sqrt[3]{w_{i j} w_{j k} w_{i k}}$ & $\sqrt{w_{i j} w_{i k}}$ \\
\hline \hline
\end{tabular}

From this, one can define the evolution upon addition of an edge $(i, v)$ of weight $\epsilon$ as

$$
\begin{aligned}
C_{i}^{O^{\prime}} & =C_{i}^{\mathrm{bin}^{\prime}} \overline{I_{\Delta i j k}^{O}}{ }^{\prime} \\
& =\frac{n_{\Delta}^{\prime}}{d_{i}\left(d_{i}+1\right)} \frac{n_{\Delta} \overline{I_{\Delta i j k}^{O}}+O(\epsilon)}{n_{\Delta}^{\prime}} \\
& =\frac{n_{\Delta}}{d_{i}\left(d_{i}+1\right)} \overline{I_{\Delta i j k}^{O}}+O(\epsilon) \\
& =\frac{d_{i}-1}{d_{i}+1} C_{i}^{O}+O(\epsilon) .
\end{aligned}
$$

\section{Directed versions of the clustering coefficients}

To generalize the Zhang-Horvath definition of clustering [12] for directed graphs, we use the same approach as proposed by Fagiolo [19]. These definitions are visible in Table VII together with the directed definitions associated with Barrat et al. [10] and Onnela et al. [11], respectively defined in Refs. [20] and [19].

\section{APPENDIX D: CLOSURE}

Closure was introduced in Ref. [13] as a complementary measure of clustering for binary undirected networks.

\section{Undirected weighted closure}

From the Zhang-Horvath definition of clustering, closure can be generalized in a fully weighted but noncontinuous way as

$$
H_{i}^{0}=\frac{\sum_{j \neq k} w_{i j} w_{j k} w_{k i}}{\sum_{j \neq k \neq i} w_{i j} w_{j k}}=\frac{W_{i i}^{3}}{\sum_{j} w_{i j}\left(s_{j}-w_{i j}\right)},
$$

again comparing the triangle intensities with their maximum possible value if all their second neighbors are also connected to them (i.e., also first neighbors) by an edge of weight 1 .

TABLE VI. Comparison of the interpretations of the different clustering definitions for undirected networks.

\begin{tabular}{lllcc}
\hline \hline Definition & Barrat & Onnela & Zhang-Horvath & Continuous \\
\hline \multirow{2}{*}{ Formula } & $\overline{w_{i}^{\Delta}} C_{i}^{\text {bin }}$ & $\overline{I_{\Delta i j k}^{O}} C_{i}^{\text {bin }}$ & $\overline{I_{\Delta i j k}^{Z}} C_{i}^{\text {bin }}$ & $\overline{I_{\Delta i j k}^{2}} C_{i}^{\text {bin }}$ \\
\hline \hline
\end{tabular}


TABLE VII. Definitions of the Barrat, Onnela, and Zhang-Horvath intensities for each partial mode pattern in directed graphs. Column 1, pattern name; column 2, Onnela triangle intensity for node $i$; column 3, Barrat triangle intensity for node $i$; column 4, Barrat triplet intensity for node $i$; column 5, Zhang-Horvath triangle intensity for node $i$; column 6, Zhang-Horvath triplet intensity for node $i$. The clustering coefficients associated with each mode $m$ are given by $C_{i}^{O,(m)}=I_{\Delta, i}^{O,(m)} / n_{T, i}^{(m)}$ for the Onnela method, $C_{i}^{B,(m)}=I_{\Delta, i}^{B,(m)} / I_{T, i}^{B,(m)}$ for the Barrat method, and $C_{i}^{Z,(m)}=I_{\Delta, i}^{Z,(m)} / I_{T, i}^{Z,(m)}$ for the Zhang-Horvath method. Note that, for the Barrat method, the reciprocal strength has been defined in Ref. [20] as $s_{i, \leftrightarrow}^{B}=\sum_{i \neq j} \frac{1}{2}\left(w_{i j}+w_{j i}\right)$.

\begin{tabular}{|c|c|c|c|c|c|}
\hline Mode & $I_{\Delta, i}^{O,(m)}$ & $I_{\Delta, i}^{B,(m)}$ & $I_{T, i}^{B,(m)}$ & $I_{\Delta, i}^{Z,(m)}$ & $I_{T, i}^{Z,(m)}$ \\
\hline Cycle & $\left(W^{\left[\frac{1}{3}\right]}\right)_{i i}^{3}$ & $\frac{1}{2}\left(W A^{2}+\left(W A^{2}\right)^{T}\right)_{i i}$ & $\frac{1}{2}\left(s_{i, \text { in }} d_{i, \text { out }}+s_{i, \text { out }} d_{i, \text { in }}\right)-s_{i, \leftrightarrow}^{B}$ & $(W)_{i i}^{3}$ & $s_{i, \text { in }} s_{i, \text { out }}-s_{i, \leftrightarrow}^{[2]}$ \\
\hline Middleman & $\left(W^{\left[\frac{1}{3}\right]} W^{\left[\frac{1}{3}\right] T} W^{\left[\frac{1}{3}\right]}\right)_{i i}$ & $\frac{1}{2}\left(W A^{T} A+W^{T} A A^{T}\right)_{i i}$ & $\frac{1}{2}\left(s_{i, \text { in }} d_{i, \text { out }}+s_{i, \text { out }} d_{i, \text { in }}\right)-s_{i, \leftrightarrow}^{B}$ & $\left(W W^{T} W\right)_{i i}$ & $s_{i, \text { in }} s_{i, \text { out }}-s_{i, \leftrightarrow}^{[2]}$ \\
\hline Fan in & $\left(W^{\left[\frac{1}{3}\right] T}\left(W^{\left[\frac{1}{3}\right]}\right)^{2}\right)_{i i}$ & $\frac{1}{2}\left(W^{T}\left(A+A^{T}\right) A\right)_{i i}$ & $s_{i, \text { in }}\left(s_{i, \text { in }}-1\right)$ & $\left(W^{T} W W\right)_{i i}$ & $\left(s_{i, \text { in }}\right)^{2}-s_{i, \text { in }}^{[2]}$ \\
\hline Fan out & $\left(\left(W^{\left[\frac{1}{3}\right]}\right)^{2} W^{\left[\frac{1}{3}\right] T}\right)_{i i}$ & $\frac{1}{2}\left(W\left(A+A^{T}\right) A^{T}\right)_{i i}$ & $s_{i, \text { out }}\left(s_{i, \text { out }}-1\right)$ & $\left(W W W^{T}\right)_{i i}$ & $\left(s_{i, \text { out }}\right)^{2}-s_{i, \text { out }}^{[2]}$ \\
\hline
\end{tabular}

This is equivalent to comparing with the situation where all open paths of length 2 are closed into a triangle by an edge of weight 1.

Finally, it can also be defined in a continuous way as

$$
H_{i}=\frac{\sum_{j \neq k} \sqrt[3]{w_{i j} w_{j k} w_{k i}^{2}}}{\sum_{j \neq k \neq i} \sqrt{w_{i j} w_{j k}}}=\frac{\left(W^{\left[\frac{2}{3}\right]}\right)_{i i}^{3}}{\sum_{j}\left(W^{\left[\frac{1}{2}\right]}\right)_{i j}^{2}-s_{i}} .
$$

\section{Directed weighted closure}

For directed networks, in contrast to Ref. [29], we only consider the extension of the undirected measure to directed paths (that is, we consistently consider only second in-neighbors via in-neighbors and second out-neighbors via out-neighbors, but not the out-neighbors of the node's inneighbors or vice versa). Therefore we define only four variants of the directed closure, two for outgoing paths [cycle out (CO) and fan out (FO)] and two for incoming paths [cycle in (CI) and fan in (FI)].

We define the weighted version either directly using the weights, as in the Zhang-Horvath definition for the clustering coefficient,

$$
\begin{aligned}
& H_{i, \mathrm{CO}}^{0}=\frac{\sum_{j \neq k} w_{i j} w_{j k} w_{k i}}{\sum_{j \neq k \neq i} w_{i j} w_{j k}}=\frac{W_{i i}^{3}}{\sum_{j} w_{i j}\left(s_{j, \text { out }}-w_{i j}\right)}, \\
& H_{i, \mathrm{CI}}^{0}=\frac{\sum_{j \neq k} w_{k j} w_{j i} w_{i k}}{\sum_{j \neq k \neq i} w_{k j} w_{j i}}=\frac{\left(W^{T}\right)_{i i}^{3}}{\sum_{j} w_{j i}\left(s_{j, \text { in }}-w_{j i}\right)}, \\
& H_{i, \mathrm{FO}}^{0}=\frac{\sum_{j \neq k} w_{i j} w_{j k} w_{i k}}{\sum_{j \neq k \neq i} w_{i j} w_{j k}}=\frac{\left(W^{2} W^{T}\right)_{i i}}{\sum_{j} w_{i j}\left(s_{j, \mathrm{out}}-w_{i j}\right)}, \\
& H_{i, \mathrm{FI}}^{0}=\frac{\left(W^{T} W^{2}\right)_{i i}}{\sum_{j \neq k} w_{k j} w_{j i} w_{k i}}=\frac{\left(w_{k j} w_{j i}\right.}{\sum_{j} w_{j i}\left(s_{j, \text { in }}-w_{j i}\right)},
\end{aligned}
$$

TABLE VIII. Some characteristic properties of the mesoscale mouse brain. SD, standard deviation.

\begin{tabular}{lccccc}
\hline \hline & Mean & SD & Median & Min & Max \\
\hline In-degree & 153.7 & 25.6 & 152 & 104 & 223 \\
Out-degree & 153.7 & 77.6 & 151 & 14 & 357 \\
CC $_{\text {tot }}^{\text {bin }}$ & 0.43 & 0.02 & 0.43 & 0.38 & 0.49 \\
Weight & 0.076 & 0.36 & 0.038 & $3.9 \times 10^{-16}$ & 20.4 \\
\hline \hline
\end{tabular}

or via the continuous definition,

$$
\begin{aligned}
& H_{i, \mathrm{CO}}^{c}=\frac{\sum_{j \neq k} \sqrt[3]{w_{i j} w_{j k} w_{k i}}}{\sum_{j \neq k \neq i} \sqrt{w_{i j} w_{j k}}}=\frac{\left(W^{\left[\frac{2}{3}\right]}\right)_{i i}^{3}}{\sum_{j}\left(W^{\left[\frac{1}{2}\right]}\right)_{i j}^{2}-s_{i, \mathrm{out}}}, \\
& H_{i, \mathrm{CI}}^{c}=\frac{\sum_{j \neq k} \sqrt[3]{w_{k j} w_{j i} w_{i k}}}{\sum_{j \neq k \neq i} \sqrt{w_{k j} w_{j i}}}=\frac{\left(W^{\left[\frac{2}{3}\right], T}\right)_{i i}^{3}}{\sum_{j}\left(W^{\left[\frac{1}{2}, T\right]}\right)_{i j}^{2}-s_{i, \text { in }}}, \\
& H_{i, \mathrm{FO}}^{c}=\frac{\sum_{j \neq k}{\sqrt[3]{w_{i j} w_{j k} w_{k i}}}^{2}}{\sum_{j \neq k \neq i} \sqrt{w_{i j} w_{j k}}}=\frac{\left(W^{\left[\frac{2}{3}\right]^{2}} W^{\left[\frac{2}{3}\right], T}\right)_{i i}}{\sum_{j}\left(W^{\left[\frac{1}{2}\right]}\right)_{i j}^{2}-s_{i, \text { out }}} \\
& H_{i, \mathrm{FI}}^{c}=\frac{\sum_{j \neq k} \sqrt[3]{w_{k j} w_{j i} w_{k i}}}{\sum_{j \neq k \neq i} \sqrt{w_{k j} w_{j i}}}=\frac{\left(W^{\left[\frac{2}{3}\right], T} W^{\left[\frac{2}{3}\right]^{2}}\right)_{i i}}{\sum_{j}\left(W^{\left[\frac{1}{2}, T\right]}\right)_{i j}^{2}-s_{i, \text { in }}} \text {. }
\end{aligned}
$$

\section{APPENDIX E: NETWORK GENERATION ALGORITHMS}

All networks were generated using the Neural Networks and Graphs' Topology (NNGT) library [28].

\section{Core-periphery network}

The core-periphery network in Fig. 1 contains (i) 1 central core node $(\mathrm{CCN})$, (ii) 10 outer-core nodes (OCNs), and (iii) 20 periphery nodes (PNs).

The nodes are connected as follows:

(i) The ten OCNs for a circular graph have fully reciprocal connections to their four nearest neighbors.

(ii) The OCNs all connect to the $\mathrm{CCN}$ with reciprocal connections.

The weights associated with these connections are drawn from $U(5,10)$. The connections with the PNs are as follows:

TABLE IX. Some characteristic properties of the Fediverse mesoscale network.

\begin{tabular}{lccccc}
\hline \hline & Mean & SD & Median & Min & Max \\
\hline In-degree & 21.3 & 82.6 & 4 & 0 & 2271 \\
Out-degree & 21.3 & 77.8 & 5 & 0 & 2038 \\
CC $_{\text {tot }}^{\text {bin }}$ & 0.68 & 0.37 & 0.86 & 0 & 1 \\
Weight & 0.045 & 0.14 & 0.0029 & $6.0 \times 10^{-7}$ & 1 \\
\hline \hline
\end{tabular}



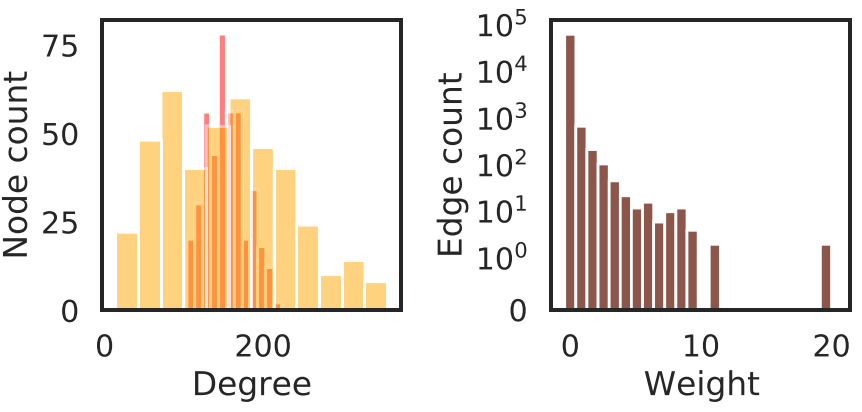

FIG. 6. Distribution of in- and out-degrees (in orange and yellow, respectively) and weights in the mouse connectome.

(i) Each OCN receives one connection from every other PN, starting with the first or second PN depending on the OCN's evenness. 0.5 .

(ii) The OCN reciprocates the connections with probability

(iii) The PNs are connected among themselves following an Erdős-Rényi scheme of density 0.05 .

Weights associated with connections involving PNs are drawn from $U(0.05,0.5)$.

\section{Watts-Strogatz network}

The original Watts-Strogatz network [30] consists of a regular lattice basis (characterized by a coordination number $k$ ) that is then modified, rewiring each edge with a probability $p$. For directed networks, we used a generalization of that method implemented in NNGT which is strictly equivalent except for the fact that edges are now directed:

(1) Start from a directed regular lattice with coordination number $k$ and reciprocity $r$ (taken as 1 in this paper).

(2) Rewire each edge with probability $p$.
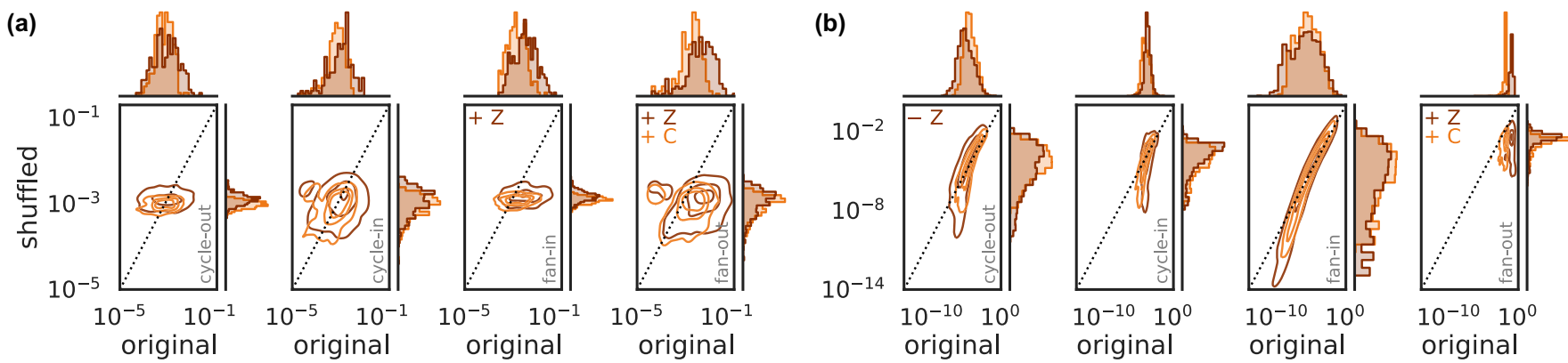

FIG. 8. Different structures of local closure in the mouse brain and Fediverse. The panels compare the local closure of directed patterns for the mouse brain (a) and Fediverse (b). The original values are compared with the averages over an ensemble of graphs with the same adjacency matrix but shuffled edge weights. For the mouse brain, 10 uniformly shuffled networks; for Fediverse, 200 shuffling across all outgoing edges of a each node (to preserve out-strength normalization). (a) The local closure also shows an increase for patterns promoting redundancy of information flow in the mouse brain (fan-in and fan-out motifs are higher in the original graph). (b) For the Fediverse, only fan out is significantly stronger than in the random graphs. Patterns where the original values are significantly greater (smaller) than the randomized ones are marked by $\mathrm{a}+(-)$ and the initial of the method (brown Z, Zhang-Horvath method; orange C, continuous method; yellow O, Onnela method). The original closure is considered to be significantly higher (lower) if $75 \%$ of the points are above (below) the dotted identity line. The addition of $*$ denotes one-sided fractions greater than $95 \%$. 
TABLE X. Number of nodes $(\mathrm{N})$ and edges $(\mathrm{E})$ and fraction of single-triangle (1-T) nodes in several collaboration and gene association networks.

\begin{tabular}{lccc}
\hline \hline Network & $\mathrm{N}$ & $\mathrm{E}$ & 1-T nodes $(\%)$ \\
\hline NetScience & 1589 & 2742 & 23 \\
CompGeo & 7343 & 11898 & 21 \\
CE-CX & 15229 & 245952 & 8 \\
CE-HT & 2617 & 2985 & 2 \\
HS-HT & 2570 & 13691 & 7 \\
SC-HT & 2084 & 63027 & 5 \\
\hline \hline
\end{tabular}

\section{APPENDIX F: REAL-WORLD NETWORKS}

\section{Mouse mesoscale connectome}

The mouse mesoscale connectome network was obtained from Ref. [22] and contains 426 nodes corresponding to brain regions of intermediate scale connected by 65465 edges. It is a symmetric version of the original network that separates nodes from both hemispheres. Each node is associated with a "name" property composed of an abbreviated denomination for the corresponding brain region, as well as a suffix (left or right) corresponding to the hemisphere. Edges are associated with three attributes: a "weight," a " $p$ value," and a "distance" (corresponding to the Euclidean distance from the source to the target node). See Table VIII and Fig. 6.

\section{Fediverse mesoscale network}

The Fediverse mesoscale network was obtained from Ref. [25] (data are available [26]). It contains 3825 nodes representing servers (instances) that are connected via 81371 edges. Each node is associated with two attributes: a "name", corresponding to the server domain, and a "size", $S_{i}$, corre- sponding to the number of users registered on that server. Edges are associated with four attributes: "num follows", defined as $F_{i j}$, the number of followers from $i$ to $j$; "follow/size", defined as $F_{i j} / S_{i}$; a "weight" given by $w_{i j}=F_{i j} / F_{i}$, the ratio between the number of followers from $i$ to $j$ divided by the total number of followers from $i$; and a "distance" giving the Euclidean distance between the two servers on the latitudelongitude plane. See Table IX and Fig. 7.

\section{Closure in the shuffled networks}

The results obtained for the local closure confirm those obtained using the clustering coefficient for the mouse brain and Fediverse networks, as shown in Fig. 8. Namely, we see an overexpression of fan-in and fan-out patterns for the mouse network and a discrepancy between those two patterns for the Fediverse network.

\section{Networks with a high number of single-node triangles}

The presence of nodes participating in a single triangle is frequent in very sparse networks such as collaboration or gene-protein interaction networks.

Table $\mathrm{X}$ details several such networks: NetScience involves coauthorship in the network science community [31], CompGeo involves collaborations in computational geometry [32], $C E-C X$ is a graph of gene associations inferred from the coexpression pattern of two genes (based on high-dimensional gene expression data) for Caenorhabditis elegans, CE-HT is for gene associations inferred from high-throughput protein-protein interactions for $C$. elegans, $H S-H T$ is for gene associations inferred from high-throughput protein-protein interactions for Homo sapiens, and $S C-H T$ is for gene associations inferred from high-throughput proteinprotein interactions for Saccharomyces cerevisiae.

Gene functional association networks [33] were downloaded [34].
[1] O. Mason and M. Verwoerd, Graph theory and networks in biology, IET Syst. Biol. 1, 89 (2007).

[2] S. E. Ahnert and T. M. A. Fink, Clustering signatures classify directed networks, Phys. Rev. E 78, 036112 (2008).

[3] X.-J. Zhang, B. Gu, X.-M. Guan, Y.-B. Zhu, and R.-L. Lv, Cascading failure in scale-free networks with tunable clustering, Int. J. Mod. Phys. C 27, 1650093 (2016).

[4] Y.-W. Li, Z.-H. Zhang, D. Fan, Y.-R. Song, and G.-P. Jiang, Influence of clustering on network robustness against epidemic propagation, in Science of Cyber Security, Lecture Notes in Computer Science, edited by F. Liu, S. Xu, and M. Yung (Springer International, Cham, Switzerland, 2018), pp. 19-33.

[5] S. F. Muldoon, E. W. Bridgeford, and D. S. Bassett, Smallworld propensity and weighted brain networks, Sci. Rep. 6, 22057 (2016).

[6] J.-G. Young, G. T. Cantwell, and M. E. J. Newman, Bayesian inference of network structure from unreliable data, J. Complex Networks 8, cnaa046 (2021).

[7] R. Guimerà and M. Sales-Pardo, Missing and spurious interactions and the reconstruction of complex networks, Proc. Natl. Acad. Sci. USA 106, 22073 (2009).
[8] M. D. Fox and M. Greicius, Clinical applications of resting state functional connectivity, Front. Syst. Neurosci. 4, 19 (2010).

[9] J. G. Orlandi, O. Stetter, J. Soriano, T. Geisel, and D. Battaglia, Transfer entropy reconstruction and labeling of neuronal connections from simulated calcium imaging, PLoS One 9, e98842 (2014).

[10] A. Barrat, M. Barthelemy, R. Pastor-Satorras, and A. Vespignani, The architecture of complex weighted networks, Proc. Natl. Acad. Sci. USA 101, 3747 (2004).

[11] J.-P. Onnela, J. Saramäki, J. Kertész, and K. Kaski, Intensity and coherence of motifs in weighted complex networks, Phys. Rev. E 71, 065103(R) (2005).

[12] B. Zhang and S. Horvath, A general framework for weighted gene co-expression network analysis, Stat. Appl. Genet. Mol. Biol. 4, 17 (2005).

[13] H. Yin, A. R. Benson, and J. Leskovec, The local closure coefficient: A new perspective on network clustering, in Proceedings of the Twelfth ACM International Conference on Web Search and Data Mining (Association for Computing Machinery, Melbourne, 2019), pp. 303-311. 
[14] J. Saramäki, M. Kivelä, J.-P. Onnela, K. Kaski, and J. Kertész, Generalizations of the clustering coefficient to weighted complex networks, Phys. Rev. E 75, 027105 (2007).

[15] Y. Wang, E. Ghumare, R. Vandenberghe, and P. Dupont, Comparison of different generalizations of clustering coefficient and local efficiency for weighted undirected graphs, Neural Comput. 29, 313 (2016).

[16] G. Kalna and D. J. Higham, Clustering coefficients for weighted networks, in AISB'06: Adaptation in Artificial and Biological Systems, edited by T. Kovacs and J. A. R. Marshall (Society for the Study of Artificial Intelligence and the Simulation of Behaviour, Bath, UK, 2006), Vol. 3, pp. 132-138.

[17] P. Holme, S. M. Park, B. J. Kim, and C. R. Edling, Korean university life in a network perspective: Dynamics of a large affiliation network, Phys. A (Amsterdam) 373, 821 (2007).

[18] K. Miyajima and T. Sakuragawa, Continuous and robust clustering coefficients for weighted and directed networks, arXiv:1412.0059 [physics.soc-ph].

[19] G. Fagiolo, Clustering in complex directed networks, Phys. Rev. E 76, 026107 (2007).

[20] G. Clemente and R. Grassi, Directed clustering in weighted networks: A new perspective, Chaos, Solitons Fractals 107, 26 (2018).

[21] I. E. Antoniou and E. T. Tsompa, Statistical analysis of weighted networks, Discrete Dyn. Nat. Soc. 2008, 375452 (2008).

[22] S. W. Oh, J. A. Harris, L. Ng, B. Winslow, N. Cain, S. Mihalas, Q. Wang, C. Lau, L. Kuan, A. M. Henry, M. T. Mortrud, B.
Ouellette, T. N. Nguyen, S. A. Sorensen, C. R. Slaughterbeck, W. Wakeman, Y. Li, D. Feng, A. Ho, E. Nicholas et al., A mesoscale connectome of the mouse brain, Nature (London) 508, 207 (2014).

[23] https://fediverse.party/.

[24] https://en.wikipedia.org/wiki/Fediverse\#Communication_ protocols_used_in_the_fediverse.

[25] M. Zignani, C. Quadri, S. Gaito, H. Cherifi, and G. P. Rossi, The footprints of a "mastodon": How a decentralized architecture influences online social relationships, in IEEE Conference on Computer Communications Workshops 2019 (IEEE, Piscataway, NJ, 2019), pp. 472-477.

[26] https://dataverse.mpi-sws.org/dataset.xhtml?persistentId=doi: 10.5072/FK2/AMYZGS.

[27] https://joinmastodon.org/.

[28] T. Fardet, NNGT 2.5.1, 2021, doi: 10.5281/zenodo.5571901.

[29] H. Yin, A. R. Benson, and J. Ugander, Measuring directed triadic closure with closure coefficients, Network Sci. 8, 551 (2020).

[30] D. J. Watts and S. H. Strogatz, Collective dynamics of 'smallworld' networks, Nature (London) 393, 440 (1998).

[31] https://networkrepository.com/netscience.php.

[32] http://vlado.fmf.uni-lj.si/pub/networks/data/collab/geom.htm.

[33] A. Cho, J. Shin, S. Hwang, C. Kim, H. Shim, H. Kim, H. Kim, and I. Lee, WormNet v3: A network-assisted hypothesisgenerating server for Caenorhabditis elegans, Nucleic Acids Res. 42, W76 (2014).

[34] https://networkrepository.com/bio.php. 